\title{
Gesundheitserziehung - die Ver(sozial)wissenschaftlichung der Gesundheitsaufklärung in den 1950er und 1960er Jahren
}

\author{
Christian Sammer
}

Health Education and Its (Social) Scientization in the 1950s and 1960s

\begin{abstract}
The contemporary history of prevention is booming. By means of its preventative societal handling of health and illness, it illuminates exemplarily the transformation in concepts, institutions, and practice of the biopolitical governing of populations. In this regard health education is a decisive technology. However, up until now the history of health education, especially with regard to its methodological transformation during this period, as well as the relations between international developments and national adaption, has not been well studied. This gap will be filled to a certain extent by means of quantitative and qualitative analysis of three different anglophone journals. During the 1950s an international professional discourse was established, which constituted a scientific occupational area. This clearly required moving away from the traditional German model of a demonstrative mediation of medical knowledge, mainly through exhibitions, and to focus on the integration of knowledge and methodology from the social sciences. From the perspective of the Behavioural Sciences human action and the possibility of it being influenced were integrated within a network of mutual influencing factors: apparently only survey research on health attitudes and motivation as well as on its own efficacy could enable the health educators to successfully understand and change human behaviour-the prioritized purpose of health education. Through such a determination of purpose, means, and relevant knowledge, which was differently received and institutionalized in the FRG as well as in the GDR, the actors of the discourse assured themselves to be part of an international profession of health educators. However, more research and the implementation of their results did not fulfill their hopes of finding "recipes for steering human" behaviour.
\end{abstract}

Keywords: Health Education, Scientization, Theories of Behaviour, Contemporary History, Transfer of knowledge, Professionalization

Die Zeitgeschichte der Prävention hat Konjunktur. An ihr lässt sich nämlich exemplarisch der Wandel von Konzepten, Organisation und Praxis des biopolitischen Regierens von Bevölkerung anhand des vorbeugenden gesellschaftlichen Umgangs mit Gesundheit und Krankheit beleuchten. Eine entscheidende Technologie ist hierbei die Gesundheitsaufklärung. Diese ist jedoch nach wie vor gerade im Hinblick auf ihre methodologischen Wandlungsprozesse sowie auf die Relationen zwischen internationalen Entwicklungen und nationalen Implementationen wenig erforscht. Auf Grundlage einer quantitativen sowie qualitativen Analyse dreier englischsprachiger Zeitschriften wird diese Lücke im Folgenden ein Stück weit geschlossen. Es wird gezeigt, wie sich in den 1950er Jahren ein internationaler Fachdiskurs der Gesundheitserziehung etablierte und in diesem sich ein verwissenschaftlichendes Berufsfeld konstituierte. Darin wurde die Forderung deutlich, vom deutschen Modell einer anschaulichen Vermittlung medizinischen Wissens vor allem durch Ausstellungen abzukommen und sich auf die Integration sozialwissenschaftlicher Erkenntnisse und Methoden zu fokussieren. Aus der Perspektive der behavioural sciences wurden das Handeln und die Möglichkeit seiner Beeinflussung in Netze wechselseitiger Einflussfaktoren eingefügt: Nur Umfrageforschung zu Gesundheitseinstellungen und -motivationen sowie zur Wirkung der eigenen Aktivitäten schien es zu ermöglichen, den in der Gesundheitserziehung priorisierten Zweck zu erfüllen, Verhalten zu ändern. Über solch eine Zweck-, Mittel- und Wissensbestimmung, die - gleichwohl unterschiedlich - sowohl in der Bundesrepublik als auch in der DDR rezipiert und institutionalisiert wurde, vergewisserten sich die Akteure im Diskurs, einer internationalen Profession der health educators anzugehö- 
ren. Doch die Hoffnungen, „Rezepte für die Lenkung der Menschen” zu finden, erfüllten sich auch mit mehr Forschung und der Implementation ihrer Ergebnisse nicht.

Schlüsse/wörter: Gesundheitserziehung, Verwissenschaftlichung, Handlungstheorien, Zeitgeschichte, Wissenstransfer, Professionalisierung

\section{Einleitung: Auf moderne Weisen Gesundheit machen}

Der moderne Mensch ist in der Regel nicht fähig, sich aus eigener Kraft und Kenntnis mit all den [...] gesundheitsrelevanten Umwelteinflüssen ohne kompetente Hilfe auseinanderzusetzen. [...] Aus diesem Grund hat sich [...] das Fachgebiet der Gesundheitserziehung entwickelt. [...] Gesundheitserziehung versucht, mit modernen Methoden der Pädagogik, der Werbung und der sonstigen Beeinflussung dem noch gesunden Menschen Verhaltensweisen näherzubringen, die sich auf die Erhaltung seiner Gesundheit positiv auswirken. [...] Wenn auch die Zielsetzungen der Gesundheitserziehung nicht heutigen Datums sind, so müssen doch ihre Methoden immer modern [...] gehalten werden $[\ldots]$. Die thematische und psychologische Vorbereitung gesundheitserzieherischer Aktionen muß durch Ergebnisse angewandter Forschung wissenschaftlich unterbaut werden. Eine empirische Basis für erfolgreiche Gesundheitserziehung setzt Forschungsmittel voraus, die nicht nur eine Beobachtung der Entwicklung anderer Forschungsund Lehrgebiete ermöglicht, sondern auch Feldstudien, Experimente und Modellversuche. ${ }^{1}$

Als der Leiter des Kölner Gesundheits-Museums Wolfgang Fritsche 1965 Aufgaben und Struktur einer zu errichtenden Bundesbehörde für Gesundheitsaufklärung skizzierte, legte er Wert auf Modernität. ${ }^{2}$ Die Entscheidung, auf dem Feld der Gesundheitsaufklärung in der Bundesrepublik neue Strukturen zu schaffen, war damals bereits getroffen. Nicht nur der Contergan-Skandal 1961/62 (vgl. Steinmetz 2003), sondern auch der Bericht des britischen Royal College of Physicians (RCP) 1962 sowie der Report of the Advisory Committee to the Surgeon General of the United States (Terry-Report) hatten die politische Bedeutung von Gesundheit und Gesundheitsvorsorge maßgeblich vor Augen geführt (vgl. Deutscher Bundestag 24.01.1964: 5027-5029; Deutscher Bundestag 11.03.1965: 8607; Berridge 2006). Die Autor*innen beider Berichte sahen das hohe Risiko des Rauchens für die Entstehung von Krebs und Herz-Kreislauf-Erkrankungen als epidemiologisch so gesichert an, dass sie nachdrücklich gesundheitspolitische Maßnahmen verlangten. Ebenso war in der Bundesrepublik die 
lange Zeit ungeklärte Frage nach der Zuständigkeit für Gesundheitsaufklärung entschieden worden: 1964 hatte die Gesundheitsministerkonferenz die Bundesregierung darum gebeten, das Kölner Gesundheits-Museum als Zentralinstitut für Gesundheitserziehung in „geeigneter Rechtsform zu übernehmen und die notwendige Finanzierung aus dem Bundeshaushalt zu ermöglichen. ${ }^{\text {3 }}$ Mit dem Anspruch, ein eigenes Fachgebiet darzustellen, forderte nun Fritsche 1965 von der Bundesregierung, eine umfassende Modernisierung der Gesundheitsaufklärung zu unterstützen. Für ihn bedeutete dies, Aufklärungsmaßnahmen explorativ zu begründen und vorzubereiten, systematisch zu planen und durchzuführen sowie ihre Wirkung gemessen am Ziel der krankheitspräventiven, individuellen Verhaltensänderung empirisch-evaluativ zu beurteilen.

Die Gesundheitsaufklärung im geteilten Deutschland ist bislang vor allem aus drei Perspektiven betrachtet worden. Zum einen liegen Arbeiten $\mathrm{zu}$ ihrer Mediengeschichte vor. ${ }^{4}$ Zum anderen thematisiert beispielsweise Rosemary Elliot das Erbe des Nationalsozialismus als dafür bestimmend, in der öffentlichen Kommunikation über Gesundheit und die Bedingungen ihrer Erhaltung politische Freizügigkeit zu demonstrieren (Elliot 2010; Elliot 2012; Elliot 2015). Vorherrschend ist aber insbesondere eine Geschichtsschreibung, die der Narration der Zeitgenoss*innen folgt (Ruckstuhl 2011). In dieser Lesart wird Gesundheitsaufklärung tendenziell abgewertet, da sie sich nicht den sozialen Bedingungen von Gesundheit und gesundem Verhalten - den Verhältnissen - zuwende, sondern am Epiphänomen des Handelns ansetze. Prägend für diese Interpretation ist die Annahme einer Marginalisierung sozialmedizinischer Perspektiven auf und Interventionsformen in das Krankheitsgeschehen zugunsten biomedizinischer Konzepte und Ansätze der Krankheitsverhütung (beispielsweise Vorsorgeuntersuchungen und -eingriffe) (Niehoff \& Schrader 1991; Schmacke 2002). So stimmig diese Interpretation auch in vielerlei Hinsicht ist, so blendet sie zweierlei aus: die (Wieder-)Etablierung von Medizinsoziologie, Sozialmedizin und Gesundheitswissenschaften als akademische Disziplinen ab den 1960er Jahren und damit die gegenläufigen Entwicklungen zur Hegemonie des biomedizinischen Paradigmas (Labisch \& Woelk 1998: 72-76; Schwartz 1995). Zudem verstellt diese Narration den Blick auf die Prozesse der Verwissenschaftlichung des Verhaltens und dessen Beeinflussung - und damit auf die einsetzende Thematisierung und Beforschung der sozialen Determinanten des Handelns. Ebenfalls kappt diese Geschichtserzählung die Verbindungen zur synchron stattfindenden politischen Aufwertung der staatlichen Verantwortung für die Gesundheit seiner Bürger (vgl. Stoff 2015).

Es steht noch aus, die Entwicklung der Gesundheitsaufklärung in beiden deutschen Staaten zu anderen nationalen Fällen zu situieren und zur inter- 
nationalen Entwicklung der Disziplin und Praxis der Gesundheitsaufklärung in Beziehung zu setzen. So haben Virginia Berridge und Luc Berlivet die Verschiebungen der Gesundheitsaufklärung zwischen 1950 und 1980 in Großbritannien und Frankreich bereits als Modernisierung beschrieben. Beide betonen ihren gestiegenen Stellenwert im Netz von Wissenschaft, breiter Öffentlichkeit und Politik. Im Zuge der Pointierung ihres professionellen Ziels, Verhaltensänderungen zu bewirken, verabschiedeten sich die Gesundheitsaufklärer*innen vom Glauben an eine handlungsleitende Einsicht aus anschaulich gemachtem biologischen und hygienischen Wissen um Gesundheit und Krankheit. Ab den 1950er Jahren rückte gerade das individuelle Handeln ins Augenmerk der Krankheitsprävention. Grundlage dafür war, dass das Risikofaktorenmodell den Weg zu weisen versprach, wie die virulenten und nunmehr als chronisch gedachten Krankheiten der Zeit, maligne Tumore und Erkrankungen des Herz-Kreislaufsystems, zu verhüten waren. Es sei frühzeitig bei den auslösenden Faktoren, das heißt Verhaltensweisen wie beispielsweise dem Rauchen, zu intervenieren. ${ }^{5}$ Die Konzepte des Risikos und der Chronizität von Erkrankungen werteten Gesundheitsaufklärung als vielversprechende Technologie einer präventiv operierenden biopolitischen Sozialtechnologie der Verhaltensbeeinflussung auf (konzeptionell: Gastaldo 1997). Werbefachleute brachten zudem die Erkenntnis ein, dass eine Wissensvermittlung nicht ausreiche, um über eine massenmediale Kommunikation das Verhalten der Einzelnen effektiv zu formen. Stattdessen sollten Medialisierungsformen und -stile aus der Werbewirtschaft individuelle Stimmungen und Gefühle sowie kollektive Wertvorstellungen beeinflussen - Faktoren, die als Determinanten des individuellen Verhaltens galten. ${ }^{6}$

Diese zunehmende Prägung der Gesundheitsaufklärung durch Erkenntnisse und Methoden der Verhaltenswissenschaften in den 1950er und 1960er Jahren ist Gegenstand meiner Ausführungen. Sie sind von einer Forschungsperspektive inspiriert, die den Zusammenhang von Produktion, Zirkulation und Rezeption von Wissen über das Soziale im 20. Jahrhundert zum Thema macht (Raphael 1996; Sarasin 2011). Im Wesentlichen geht es dabei darum, die Kopplungen zwischen systematischen Betrachtungsweisen gesellschaftlicher Prozesse und Interventionen in das Soziale auf der Ebene des entstehenden Wissens, der verwendeten Praktiken und der beteiligten Akteure mit ihren professionellen Strategien und Weltvorstellungen im Verlauf zu untersuchen (etwa Lengwiler 2010). ${ }^{7}$ Dass hierbei Untersuchungen zur Geschichtlichkeit des Wissens und der Techniken der Prävention als „exemplarische Kulturgeschichte der Moderne“ die Zeitgeschichte des Sozialen bereichern, ist einschlägig demonstriert worden (vgl. Lengwiler \& Madarász 2010; Schenk et al. 2013; Hähner-Rombach 2015). ${ }^{8}$ In der Zeitgeschichte der Gesundheitsaufklärung bestehen jedoch noch 
einige Forschungslücken. Eine davon betrifft den miteinander verschränkten organisatorischen, medialen und konzeptionellen Wandel der 1960er Jahre, der im begrifflichen Wechsel der Zeitgenoss*innen von hygienischer Volksbildung, -aufklärung und -belehrung zur Gesundheitserziehung zum Ausdruck kommt: Welche Programme und Methoden - die Wolfgang Fritsche 1965 eingefordert hatte - galten nunmehr als zeitgemäß, welche lösten sie ab und woher kamen diese? Wer machte sie auf welche Weise stark?

Den Antworten auf diese Fragen nähere ich mich in fünf Schritten an: Erstens skizziere ich Programm, Geschichte und Struktur der Gesundheitsaufklärung in der Bundesrepublik und der DDR. Hier geht es mir darum, anhand von zeitgenössischen Publikationen und Archivmaterial aus dem Deutschen Hygiene-Museum (Dresden), dem Deutschen Gesundheits-Museum (Köln) und den jeweiligen Medizinalverwaltungen aufzuzeigen, wie Gesundheitsaufklärung in beiden deutschen Staaten bis in die 1960er Jahre organisiert war und mit welchen Konzepten und Medien sie operierte. Anhand dieser Quellen wird, zweitens, deutlich, dass dieses „Dresdner Modell“ in den 1950er Jahren in die Kritik geriet, an die sich bis zum Ende des darauffolgenden Jahrzehnts, drittens, eine umfassende Neugliederung und -ausrichtung der Gesundheitsaufklärung in beiden deutschen Staaten anschloss. Da vor allem die geringe Wissenschaftlichkeit sowie die unzureichende Rezeption des internationalen Wissensstandes beanstandet wurde, erscheint es notwendig, den Fachdiskurs der Gesundheitsaufklärung in den 1950er und 1960er Jahre zu analysieren. Dies erfolgt zweistufig: Im vierten Schritt werden drei anglofone Fachzeitschriften zuerst einer quantitativen Inhaltsanalyse unterzogen, in denen Gegenstände und Methoden der Gesundheitserziehung (health education) als Sozialwissenschaft geordnet wurden. Anglo-amerikanische Gesellschaften bildeten und trugen diesen Diskurs maßgeblich. Aber auch internationale Organisationen wie die Weltgesundheitsorganisation (WHO) oder die International Union for Health Education of the Public (IUHEP) - ein 1951 in Paris gegründeter internationaler Zusammenschluss zur Förderung der Gesundheitsaufklärung, dem 1967 mindestens 32 nationale Komitees angehörten - spielten eine wichtige Rolle. Das vom Central Council for Health Education des Vereinigten Königreichs herausgegebene Health Education Journal erscheint seit 1943. 1950 wurde in den Vereinigten Staaten die Society for Public Health Educators als Berufsverband der Gesundheitserzieher*innen gegründet, der 1957 begann, die handlungssoziologisch ausgerichtete Zeitschrift Health Education Monographs (seit 1997 Health Education E Behavior) zu publizieren. Ein Jahr später zog die sich als „world parliament of health educators" verstehende IUHEP nach: Seit 1958 veröffentlicht die Union in enger Absprache mit der Weltgesundheitsorganisation vierteljährlich das International Journal of Health Education als offizielles Organ 
(1982-1993 unter dem Titel Hygie, bis 2008 Promotion E Education und heute unter der Bezeichnung Global Health Promotion). Sie versteht dieses explizit als eine Plattform über die sich Gesundheitserzieher*innen über ihre Programme, Methoden, Techniken, Materialien sowie Erfahrungen austauschen können. Dies geschah in den 1950er und 1960er Jahren auch auf regelmäßig stattfindenden Konferenzen (Viborel 1958b). Kriterium für die Auswahl dieser Fachjournale war, dass sie die einzigen in englischer, deutscher, französischer und russischer Sprache sind, die titelgebend einen expliziten und schwerpunktmäßigen Bezug zur Gesundheitsaufklärung aufweisen und zwischen 1950 und 1970 periodisch erschienen sind. ${ }^{9}$ Sammelbände, veröffentlichte Berichte und Bibliografien wurden von der Analyse ausgeschlossen, weil davon wenig Erkenntnis über die kontinuierliche methodologische Entwicklung der Disziplin und des Berufsfeldes der Gesundheitsaufklärung zu erwarten war. ${ }^{10}$ Das zunächst quantitative Vorgehen vermisst den Diskurs der health education und orientiert die sich, fünftens, anschließende qualitative und damit notwendigerweise verdichtende und exemplarische Auswertung seiner mehr als zehntausend Seiten. Relevanz, Häufung sowie Gewicht von bestimmten Schlagworten wurden über die Volltextsuche in den Zeitschriften bestimmt. Im Fall des International Journal of Health Education verantworte ich diese selbst, bei den beiden anderen Journalen deren heute gemeinsamer Verlag Sage Publications. Zusammenfassende Äußerungen und ausblickende Überlegungen zur weiteren Entwicklung der Gesundheitsaufklärung beschließen den Aufsatz.

\section{Das Dresdner Modell der Gesundheitsaufklärung - und seine Krise}

In Deutschland operierte die Gesundheitsaufklärung in der ersten Hälfte des 20. Jahrhunderts in einem Modus, den die Zeitgenoss*innen „hygienische Volksbildung, Volksaufklärung oder Volksbelehrung“ nannten (vgl. Hagen 1920; Vogel 1925; Straschun 1928). Eine seiner Wurzeln lag im Messe- und Ausstellungswesen des 19. und frühen 20. Jahrhunderts (vgl. Nikolow \& Brecht 2000). Aus der Initiative, die zur I. Internationalen Hygiene-Ausstellung 1911 in Dresden geführt hatte, etablierte sich das Deutsche Hygiene-Museum (DHM) in Dresden als modernes Gegenwartsmuseum und gewichtiger Akteur der Gesundheitsaufklärung (vgl. Münch \& Lazardzig 2002; Stein 2013; Heesen 2012: 87-104). Auf die finanziellen Krisen der Weimarer Republik reagierte der Leiter des Museums, der Kaufmann Georg Seiring (1883-1972), mit einer betriebswirtschaftlichen Expansions- und Diversifizierungsstrategie: Zum Ende der Weimarer Republik war dadurch das Hygiene-Museum zu einer rechtlich eigenständi- 
gen Einrichtung geworden, die von einem Verein getragen und zu großen Teilen vom Dresdner Stadtrat und den Regierungen des Landes Sachsen und des Reichs finanziert wurde. Zusätzlich waren an das DHM eine Hygieneakademie für Vortragsdienste, ein Verlag, eine Aktiengesellschaft und ein Internationaler Gesundheitsdienst angekoppelt, die je eigene Umsätze erwirtschafteten (vgl. Steller 2014). Als „Hygiene-Konzern “11 bot das Museum eine Vielzahl unterschiedlicher und sich gegenseitig ergänzender Aufklärungsmedien und -dienstleistungen an. Von 1926 bis 1931/33 gab es darüber hinaus ein eigenes Fachjournal für die hygienische Volksbelehrung, den Hygienischen Wegweiser, heraus. Entsprechend jeweiliger sozialhygienischer Problematisierungen entwarfen, präparierten, modellierten, zeichneten, klebten und bemalten die Mitarbeiter*innen des Museums eigene Exponate und verkauften serienförmige Abwandlungen an medizinische Lehreinrichtungen, Krankenhäuser, Gesundheitsämter und Schulen. Seine Wanderausstellungen tourten bis 1944 durchs Deutsche Reich und Europa (Weinert 2017). Mit ihnen sollten die Besucher*innen über die Anatomie und Physiologie (menschlicher) Körper, die Bedingungen ihrer Gesundheit und Erkrankung sowie über die Strukturen des Gesundheitswesens belehrt werden (Nikolow 2015a). Nach 1945 erkannten die Besatzungs- und zivilen Verwaltungsbehörden schnell den Nutzen einer solchen Einrichtung und verstaatlichten das international renommierte Museum 1946. Nach dem daraufhin folgenden Weggang Georg Seirings erhielt das Dresdner Modell eine deutsch-deutsche Dimension im Systemkonflikt des Kalten Kriegs, denn Seiring migrierte nach Köln, wo er 1949 das Deutsche GesundheitsMuseum in Köln gründete (Sammer 2013).

Im konzeptionellen Kern der hygienischen Volksbelehrung stand die Wirkungsannahme, dass die plastischen oder gar bedienbaren Exponate vielen Besucher*innen das Wissen der Physiologie, Anatomie und Hygiene so anschaulich präsentieren könnten, dass dieses handlungsleitend und damit verhaltenspräventiv wirkte (vgl. Vogel 1925: 303-312, 342). Die Ordnung allen Lebens und Zusammenlebens zu erkennen, würde zwangsläufig eine gesundheitsgemäße Lebensführung zum Schutz dieser Ordnung mit sich bringen (vgl. Lingner 1930 [1904]; Lingner 1914; Engelhardt \& Vogel 1930). Als ein geeignetes Medium einer solchen lebensphilosophischen sowie rationalistischen Annahme der Handlungsrelevanz von Wissensvermittlung galt die Ausstellung - das Massen- und Anschaulichkeitsmedium der Zeit.

Im Laufe der 1950er Jahre gerieten die Struktur, das Kommunikationsmittel Ausstellung sowie das pädagogische Konzept der hygienischen Volksbelehrung in beiden deutschen Staaten in die Kritik. Die Gesundheitserzieher*innen, die in beiden deutschen Staaten entweder an den $\mathrm{Mu}$ seen oder in den Medizinalverwaltungen beschäftigt waren, verwendeten 
dabei eine Rhetorik, mit welcher sie eine Rückständigkeit im Vergleich mit Frankreich, den USA oder der Sowjetunion betonten: Nur eine bessere wissenschaftliche Vorbereitung und Begleitung, eine eindeutige Ausrichtung auf Erziehung zum Handeln sowie zentrale Organe mit klaren Zuständigkeiten würden zu einer wirksameren Präventionsleistung der Gesundheitsaufklärung führen (vgl. Hünerbein 1953; Zoller 1957; Steidle 1955). ${ }^{12}$ In Anbetracht der häufig auftretenden Herz-Kreislauferkrankungen, die als Verhaltenskrankheiten gedeutet wurden, reiche eine Aufklärung über die menschliche Anatomie und Physiologie nicht mehr aus, so der Tenor. Als eine Praxis der Verhaltensänderung hatte eine inzwischen Gesundheitserziehung genannte Tätigkeit sich thematisch zu fokussieren und Zielgruppen zu differenzieren. Vor allem aber musste sie von der "gesundheitserzieherischen Aktion her entwickelt werden. ${ }^{13}$ Nicht mehr nur aufzuklären, sondern zu gesunden Lebensgewohnheiten zu erziehen, stand nun auf der Agenda der Gesundheitsaufklärung ganz oben (vgl. Kunkel 1962: 24). Das entwertete zugleich auch die etablierten Medien - Ausstellungen, Exponate und Lehrmittel - des Dresdner Modells einer hygienischen Volksbelehrung.

Die Kritik an alten, als ineffektiv geltenden Medien und Konzepten des Dresdner Modells wurde ebenfalls in der Zeitschrift Health Education geäußert. ${ }^{14}$ Die Ausstellung stelle, so war zu lesen, die größten Herausforderungen an Einfallsreichtum und Können und benötige viel Zeit und Energie. Sie sollte nur dann noch Verwendung finden, wenn ihre dreidimensionalen Exponate anderen Medien an Schauwert klar überlegen seien und die Ausstellungmacher all die notwendigen Ressourcen mobilisieren könnten (Rowntree 1959).

Aber was machte das Beurteilungskriterium der Effizienz aus, abgesehen von den Kosten? Die Antwort bestand in der Möglichkeit zur Verhaltensänderung: Der Vorteil der Ausstellung läge gerade darin, dass es der Gesundheitserziehung weniger um die Vermittlung von Wissen, sondern vielmehr um die Entwicklung von für die Gesundheit förderlichen Einstellungen (attitudes) und Verhaltensweisen (practices) ginge. Die prinzipielle Verbindbarkeit von unpersönlichen mit persönlichen Kommunikationsformen lasse die Ausstellung zu mehr werden als einem Ort der Informationsvermittlung, so die Verteidiger dieses desavouierten Mittels. Sie machten die Ausstellung zu einem Ort des Verstehens, verbunden mit spezifischen Veränderungen individueller Handlungsgewohnheiten (individual habits) (Rowntree 1959: 110). Das erreiche dieses Medium aber nur mit einer dramatischen Narration und dem Einsatz aller möglichen medialen Ergänzungen („film shows, panel discussions, quizzes, poster competitions, talks, demonstrators, visitor participation as much as possible“, Rowntree 1959: 114f.). All dies setze voraus, im Vorfeld minutiös zu planen und die 
Interessen und Bedürfnisse der anvisierten Zielgruppe zu bestimmen. An diesen sollte die Gestaltung der Ausstellung inhaltlich wie formal ausgerichtet und ihre Wirkung im Nachhinein beurteilt werden (Rowntree 1959: 117f.). Die Befürworter der Ausstellung präsentierten ihr Medium als ein sehr anspruchsvolles, aber auch zeitgemäßes, sprich als eines, das auch erziehen konnte und $\mathrm{zu}$ verwissenschaftlichen war.

Der wissenschaftliche Leiter des Hygiene-Museums und spätere Sektorenleiter für Gesundheitserziehung im Ministerium für Gesundheitswesen der DDR, der Mediziner Rolf Thränhardt, nutzte 1959 ebenfalls das Health Education Journal, um für das Hygiene-Museum in Dresden zu werben. Rolf Thränhardt erwähnte zwar auch die Stärken seines Museums die Lehrmittelproduktion und Ausstellungsgestaltung -, doch auch Thränhardt rückte etwas Anderes in den Vordergrund als die museale Tradition der hygienischen Volksbelehrung:

People must be educated out of a passive attitude and into an active attitude towards hygiene, in order to use, by individual motivation, all the possibilities for maintaining health that modern science offers. Only conscious personal action can enable them in the course of a healthy way of life to make sure of long life [...]. Medical science is thus presented with an educational problem, to be solved with the accustomed scientific exactitude. (Thränhardt 1959: 124)

Thränhardt betonte das Ziel der Verhaltensänderung, welche über die entsprechende Formung einer inneren Motivation geschehe. Besonders scharf formulierte er diese als Aufgabe einer Bewusstseinsformung, die wiederum nur mit wissenschaftlicher Methodologie und epistemischer Moral (Exaktheit) ihre Aufgaben lösen könne. Hier aber müsse das Dresdner Museum nacharbeiten: „Scientific research is the newest branch of the work of the German Hygiene-Museum. In this field there is comparatively little previous experience" (Thränhardt 1959: 126). ${ }^{15}$

Der Vertrauensverlust in die Ausstellung als geeignetes Mittel der Gesundheitsaufklärung wurde in den Zeitschriften einige Jahre später, 1962, schließlich offenkundig. Im Health Education Journal unterstrich Bruno Gebhard (1901-1985) den Nutzen eines Museums der Gesundheitsaufklärung, das eng mit dem Dresdner Modell verwandt war. Gebhard hatte von 1927 bis 1932 im Hygiene-Museum gearbeitet und hielt auch noch in den Jahren danach engen Kontakt mit dem Museum. Schließlich gelang es Gebhard in der zweiten Hälfte der 1930er Jahre das Dresdner Modell, das "class showmanship" mit "first class science“ verband, in die Vereinigten Staaten zu exportieren und dort in Form des Cleveland Health Museum zu etablieren (Calver 1962: 214). Dessen Geschäfte führte Gebhard von 1940 bis 1965. Von dort unterstützte er seinen ehemaligen Vorgesetzten Georg 
Seiring, indem er zuerst in Dresden und 1949 vom Deutschen Gesundheits-Museum in Köln Exponate für das Museum in Cleveland bestellte (Weinert 2017: 199-217).

In seinem Beitrag wählte Gebhard ein defensives Narrativ, mit dem er die Organisationsform des Gesundheitsmuseums zu rechtfertigen und sie zugleich als modern zu beschreiben versuchte. Laut Gebhard könnte es dem Fernsehen Requisiten anbieten sowie thematische Sonderausstellungen mit Bezügen zu persönlichen und familiären Angelegenheiten der Besucher präsentieren. Dafür wiederum müsste eine Technik fest etabliert werden: evaluative Verfahren, die die Einstellungen und Meinungen der Besucher sichtbar und die Wirkung der Ausstellungen auf diese greifbar machten. Nur so werde das Gesundheitsmuseum zu einer anerkannten Bildungseinrichtung; nur dadurch erlange es das Image, sich aktuellen Belangen $\mathrm{zu}$ widmen. Dann werde ein solches Museum auch nicht als ein "Mausoleum“ (Gebhard 1962: 91) wahrgenommen. Mit einem Supermarkt-Display zur Ernährung hoffte Gebhard, das Gesundheitsmuseum als ein neues, modernes Phänomen, gleichwohl ohne kommerzielle Interessen, darstellen zu können. Gebhard schrieb die regelmäßig zu erneuernden und thematisch zu fokussierenden Wechselausstellungen in die Modernität der amerikanischen Konsumgesellschaft sowie in die der Gesundheitsmuseen ein (vgl. Abb. 1).

Gebhard war zu dieser Zeit auch noch in der DDR als international erfolgreicher Volksbelehrer geschätzt. So wurde er 1961 zur Nationalen Hygiene-Ausstellung eingeladen, die zum 50-jährigen Jubiläum der I. Internationalen Hygiene-Ausstellung in Dresden stattfand. Aus seinen persönlichen Notizen wird jedoch ersichtlich, dass es entgegen seiner öffentlichen Ausführung um die Gesundheits-Museen zu dieser Zeit nicht gut bestellt war: Der Besucherzuspruch war in Cleveland wie in Dresden niedrig. ${ }^{16}$ So zog Gebhards Modernitätsrhetorik des Dresdner Modells auch aus dem Blickwinkel der Gesundheitserziehung eine entschiedene Widerrede nach sich.

Homer Calver (1892-1970), ehemaliger Generalsekretär der American Public Health Association und engagierter Befürworter der Gründung eines Health Museum nach Dresdner Vorbild in den USA in den 1930er Jahren, widersprach Bruno Gebhard entschieden. Ebenfalls einen historischen Bogen schlagend, kontrastierte er das Museum in Cleveland mit der Vielzahl an gescheiterten Versuchen, ähnliche Einrichtungen zu gründen (vgl. McLeary \& Toon 2012). Gebhards Museum ging allerding daraus nicht als Muster des Erfolgs hervor. Im Gegenteil, Calver betrachtete es als einen historischen Sonderfall (Calver 1962: 214). Er bestand darauf, ein Gesundheitsmuseum nur nach den Kriterien der Gesundheitserziehung zu beurteilen, also danach zu bewerten, inwiefern es beispielsweise 


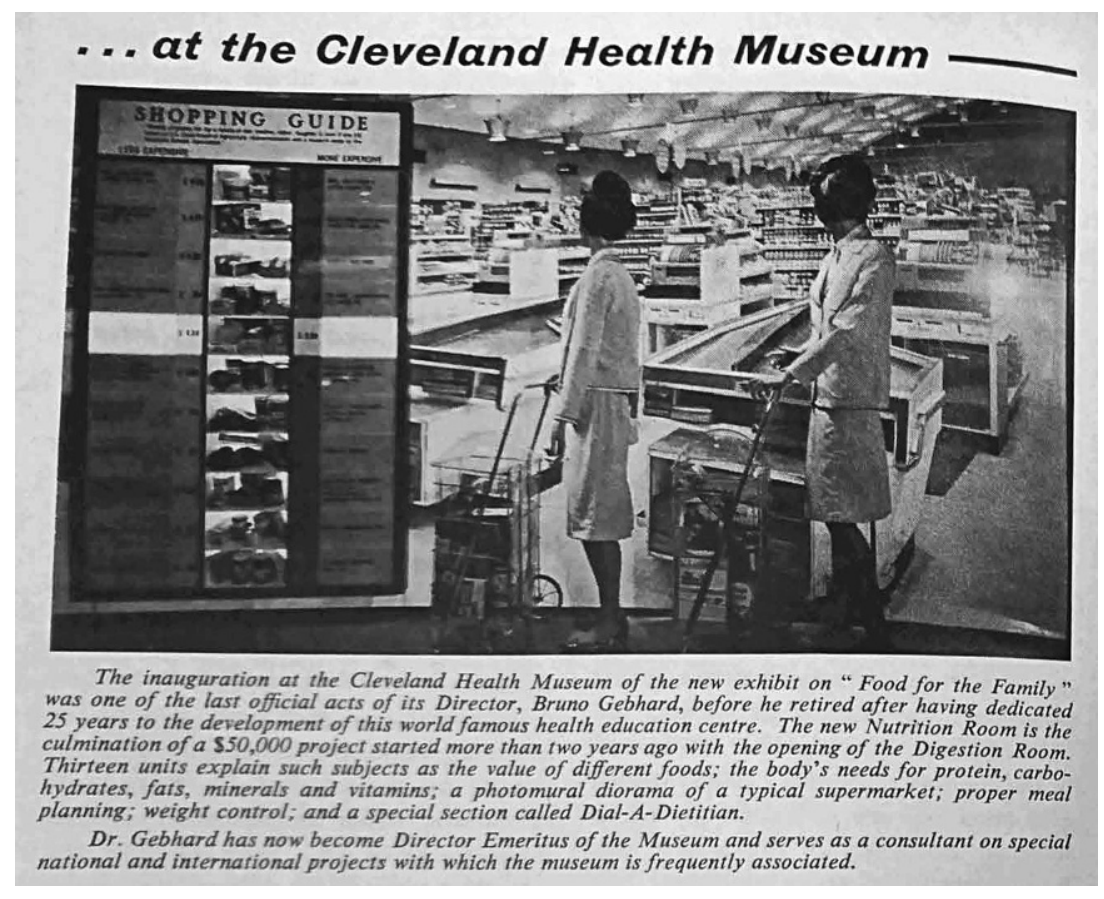

Abb. 1 Werbung für die neue Ausstellung des Cleveland Health Museum, 1965 (The Health Education Journal 1965 (8): 100)

durch eine Ernährungsausstellung dazu beitragen könne, zu „good dietary habits" (Calver 1962: 216) zu ermutigen. Nach Calvers Verständnis war der Existenzzweck eines Gesundheitsmuseums, Verhaltensgewohnheiten $\mathrm{zu}$ ändern und nicht, ein unabhängiger Lernort zu sein. Gemessen daran, könne dem aufwändigen Museum in Cleveland im Vergleich mit dem Fernsehen, dem Kinofilm und den „mass circulation media“ (Calver 1962: 216) nur die Verschwendung knapper Ressourcen vorgehalten werden. Einem Gesundheitsmuseum blieben nur zwei Möglichkeiten: entweder als eine Koordinationsstelle für Gesundheitserziehungskampagnen zu fungieren oder Forschung zur Gesundheitserziehung zu betreiben. Und mit welchen Wissensbeständen und Zugängen Letzteres unternommen werden könnte, referierte Calver knapp: „We have learned the paramount value of the approach [of health education programmes, C.S.] through the behavioural sciences" (Calver 1962: 216).

Die Museen in Cleveland, Köln und Dresden standen demzufolge allesamt vor der gleichen Problemkonstellation. Mit der abnehmenden Besucherattraktivität sank die Legitimität der Organisationsform Museum und die Gesundheitserzieher*innen erlangten Deutungshoheit. Und je stärker ihr interpretativer Zugriff, desto alternativloser wurde selbst für die Für- 
sprecher*innen von Gesundheitsausstellungen und -museen die Ausrichtung auf eine Gesundheitserziehung, die mit neuen Medien, mit Rückgriff auf die behavioural sciences und mit evaluativen Verfahren auf die Änderung von Verhalten abzielte. Im Zuge des Aufbrechens der behavioristischen Orthodoxie in den 1950er Jahren war vor allem in den Vereinigten Staaten der Begriff der behavioural sciences als Sammelbegriff für unterschiedliche disziplinäre Zugänge (etwa aus der Anthropologie, Ethnologie, Soziologie, Psychologie, Psychiatrie oder auch der Linguistik) zur Erforschung des menschlichen Verhaltens aufgekommen. Auf Bedingungen, Prozesse und Effekte der Kognition blickend verhießen diese Ansätze auch für die Gesundheitserziehung Perspektiven, um Gesundheitsverhalten in seiner sozialen Situiertheit verstehen und verändern zu können (vgl. Carlson \& Watson 1965; Bruner 1990: 4f.).

\section{Gesundheitserziehung in beiden deutschen Staaten}

Seinen institutionellen Niederschlag fand diese Verschiebung des Modus der Gesundheitsaufklärung von der hygienischen Volksbelehrung zur Gesundheitserziehung in beiden deutschen Staaten im Laufe der 1960er Jahre. In neuen oder umgewidmeten Organisationen schufen sich die Gesundheitserzieher*innen eine geeignete Infrastruktur, um Verhaltensänderung zu bewirken, ihre Praxis zu beforschen und ihre Maßnahmen zu koordinieren. In der DDR wurde 1961 ein zentrales Beratungsgremium für Gesundheitserziehung am Ministerium für Gesundheitswesen gegründet - das Komitee für gesunde Lebensführung und Gesundheitserziehung in der DDR (NKGE, ab1969 Nationales Komitee für Gesundheitserziehung der DDR). Sechs Jahre später wertete eine Umstrukturierung die wissenschaftliche Abteilung des Hygiene-Museums zu dem eigenständigen Institut für Gesundheitserziehung (IfG) unter dem Dach des DHM auf. Das Gesamtgebilde Hygiene-Museum wurde zum „leitenden Zentralinstitut auf dem Gebiet der Gesundheitserziehung und der Produktion von biologischanatomischen Unterrichtsmitteln und Anschauungsmaterialien"17 ernannt. Das NKGE gab auf dem neu strukturierten Feld der Gesundheitsaufklärung die Problemstellungen und Strategien der Gesundheitserziehung vor, das IfG übernahm als ausführendes Organ die Aufgabe, dafür Wissensgrundlagen $\mathrm{zu}$ erarbeiten und die entsprechenden Kommunikationsmedien $\mathrm{zu}$ schaffen. Ebenfalls 1967 fanden die organisatorischen Veränderungen in der Bundesrepublik ihren Abschluss: Die Bundeszentrale für gesundheitliche Aufklärung (BZgA) trat an die Stelle des Deutschen GesundheitsMuseums. Mit ihr errichtete die Bundesregierung eine bundesunmittelba- 
re Behörde im Geschäftsbereich des Ministeriums für Gesundheitswesen. Sie verlieh damit der erklärten staatlichen Verantwortlichkeit und ihrem Gestaltungswillen, für die „Erhaltung und Förderung der Gesundheit"18 $\mathrm{zu}$ sorgen, eine institutionelle Form. Grundsatz- und Richtlinienarbeit, die Aus- und Fortbildung von Gesundheitserzieher*innen, die Koordination entsprechender Maßnahmen sowie die internationale Zusammenarbeit standen auf der Agenda der BZgA. Ihre Abgrenzung zum Bundesausschuss für gesundheitliche Volksbelehrung erfolgte ein Jahr später. Der seit 1954 bestehende Freiwilligenverband, der sich nun Bundesvereinigung für Gesundheitserziehung nannte, deckte ausschließlich den „nichtbehördlichen Raum" und damit die Aufgabe der Koordination mit nicht-staatlichen Akteuren ab (vgl. Bundesvereinigung für Gesundheitserziehung e. V. 1989: 6).

In beiden Staaten entwarfen die Gesundheitserzieher*innen Schwerpunktkampagnen, die beispielsweise das Rauchen zielgruppenspezifisch thematisierten und konzertiert über Presse, Film und Rundfunk Verbreitung finden sollten. Auf beiden Seiten der innerdeutschen Grenze folgten die Akteure einer Modernitätsvorstellung ihrer Arbeit, in deren Zentrum die Forderung stand, gesunde Lebensweisen mithilfe der Erforschung der Beeinflussungsfaktoren individuellen Handelns sowie einer Wirksamkeitsbestimmung der eigenen Maßnahmen anzuerziehen: Passives Wissen sollte endlich in aktives Handeln überführt werden. ${ }^{19}$ Dafür rekrutierten die Einrichtungen in der DDR wie auch in der Bundesrepublik eine neue Generation von Beschäftigten, die ihre fachliche Sozialisation als Pädagogen, Soziologen, Psychologen oder in der öffentlichen Kommunikation erlangt hatten. Diese neuen Mitarbeiter*innen wandten die Erkenntnisse der Verhaltenswissenschaften und das Methodenset der Evaluationsforschung an oder integrierten eine solchermaßen gestaltete Expertise. Ebenfalls kodifizierten sie den Wissensbestand der Gesundheitserziehung ab dem Ende der 1960er Jahre in Form von Anleitungen, Grundrissen, Handbüchern oder Mitteilungen (vgl. Bundesvereinigung für Gesundheitserziehung e.V. 1957-1986; Ludwig 1973b).

Selbstverständlich operierte Gesundheitserziehung in der Bundesrepublik und in der DDR unterschiedlich. Zu heterogen waren die Leitbilder der Erziehung, die Medienlandschaft, gesellschaftspolitische Engführungen top down sowie der Organisationsgrad Betroffener. Auch gab es unterschiedliche Möglichkeiten, die Ergebnisse einer gesellschaftlichen Beobachtung, die den Erwartungen politischer Ideologien widersprachen, auf einem staatlich sanktionierten Kanal zu kommunizieren, auf dem beide Regierungen doch ihre Sorge um die Gesundheit ihrer Staatsbürger*innen zeigen wollten. Retrospektiv auffällig ist vor allem, dass die Bundeszentrale umfassend sozialwissenschaftliche Studien beauftragte und rezipierte, welche die Einstellungen, Motivationen, Verhaltensweisen sowie die 
Wahrnehmung und Wirkung von Kommunikationsmedien vermaßen. Deren Ergebnisse wurden in Projektentwürfen integriert und diese mit gesellschaftlichen und politischen Akteuren (Interessensvereinen oder Selbsthilfegruppen) kontrovers diskutiert. Von solchen Forschungsarbeiten sind für den Zeitraum zwischen 1968 und 1975 mehr als 120 archiviert. ${ }^{20}$ Ähnliche explizit auf Kampagnenmaterial zugeschnittene externe Expertisen lassen sich in den Hinterlassenschaften des IfG oder des NKGE nicht finden. Dort brachten die Mitarbeiter*innen beziehungsweise die Mitglieder (aus der Wissenschaft oder den gesundheitspolitischen Massenorganisationen wie dem Deutschen Roten Kreuz) ihre Erfahrungen und Erkenntnisse ein.

Gleichwohl kamen die Gesundheitserzieher*innen in der Projektentwicklung und -umsetzung zu ganz ähnlichen Ergebnissen. Dabei rekurrierten sie zwar nicht explizit auf Äußerungen aus dem internationalen Diskurs, jedoch waren ihre Annahmen, Positionen und Erkenntnisse die gleichen: Für Aufklärungskampagnen zur Beeinflussung des Rauchverhaltens beispielsweise müsste zunächst empirisch der je spezifische Sinn des Rauchens verstanden werden, bevor über gleichfalls zielgruppendifferenzierte Kommunikationskanäle sozial maßgeschneiderte und positiv gehaltene Botschaften Aussicht auf eine handlungsleitende Rezeption haben konnten. Solche Aktionen mussten selbstverständlich über ihre gesamte Laufzeit hinweg evaluativ vorbereitet, begleitet und ausgewertet werden. ${ }^{21}$

Ein zweiter markanter Unterschied zwischen der Praxis der Gesundheitserziehung in der Bundesrepublik und der DDR bestand in der Intensität, mit der sich die Gesundheitserzieher*innen am internationalen Diskurs beteiligten. Die Ausführungen von Vertreter*innen aus dem sozialistischen Deutschland zum Stand und den Erfahrungen der Gesundheitserziehung beispielsweise in den Publikationen der IUHEP erscheinen vorsichtig und schablonenhaft (vgl. exemplarisch Ludwig 1973a und Fritsche 1973 im Kontrast). Sie suchten auch vorrangig Kooperationen mit sozialistischen Partnerinstituten (vgl. exemplarisch Institute für Gesundheitserziehung der sozialistischen Länder Europas 1980). Die westdeutschen Gesundheitserzieher*innen aus der BZgA beteiligten sich dagegen umfassend am nach Westen blickenden internationalen Austausch. So veranstaltete die Bundeszentrale in Zusammenarbeit mit der IUHEP ab 1969 Internationale Seminare für Gesundheitserziehung (vgl. Bundeszentrale für gesundheitliche Aufklärung 1970). Ab 1972 gab sie gar die deutschsprachige Ausgabe des International Journals of Health Education heraus. ${ }^{22}$ In den 1970er Jahren trug die BZgA so maßgeblich den internationalen Fachdiskurs der Gesundheitserziehung mit. Ihr erster Präsident Wolfgang Fritsche, der 1965 so entschieden für mehr Forschung in der Gesundheitserziehung plädiert hatte, ging 1977 sogar als Berater, Medical Officer und internationaler Koordinator an das Europabüro der Weltgesundheitsorganisation 
(WHO) in Kopenhagen. ${ }^{23} 1983$ wurde die BZgA schließlich offizielles Kooperationszentrum des Europabüros; das DHM erlangte diesen Status 1987 (vgl. zu diesem Kapitel insgesamt: Sammer 2015: 267-279).

\section{Die Ver(sozial)wissenschaftlichung der Gesundheitsaufklärung im Modus der Gesundheitserziehung - quantitativ}

Wie wurden nun Ziele, Zwecke, Methoden und Medien der Gesundheitserziehung konkret entworfen? Woher kamen diese Konzepte? Welche sind die Schlüsselbegriffe der Gesundheitserziehung, und wo clusterten sich diese im zeitlichen Verlauf? Eine quantitative Inhaltsanalyse der drei im Volltext erschlossenen Zeitschriften nach trunkierten Stichworten liefert einige Hinweise auf Antworten (vgl. Tab. 1). Ein solcher erster Analyseschritt soll die jeweiligen Gewichtungen von bestimmten Begriffen im Verlauf abbilden. Weil der Umfang der einzelnen Zeitschriften relativ stabil bei ca. 200 bis 300 Seiten pro Jahrgang blieb, kann auf Berechnungen verzichtet werden, die in schließenden Statistiken die Validität der Schlussfolgerung bestimmen (Wahrscheinlichkeitsverteilung, Erwartungswerte, Varianz, vgl. Härtler 2014). Bei den Trefferzahlen handelt es sich um Mindestwerte, die weder eine exakte Bestimmung von Repräsentativität leisten können noch die einzelnen Begriffe nach ihrer textimmanenten Bedeutung hin unterscheiden. Mehrfache Funde innerhalb einer Texteinheit wurden einfach gezählt, die Stellung des jeweiligen Begriffs im Titel oder Fließtext nicht unterschieden. Jeder Treffer steht demnach für eine Literaturübersicht, einen Forschungsartikel meistens im Umfang von fünf bis zehn Seiten oder einen Konferenzbericht. Im Gegensatz zu dem Health Education Journal und den Health Education Monographs erstreckte sich die Suche im International Journal of Health Education außerdem auf Ankündigungen, Berichte, Meldungen, Leserbriefe und Rezensionen. Trotz dieser Einschränkungen detektiert ein solches Vorgehen gleichwohl allgemeine thematische Schwerpunkte sowie deren Entwicklung und orientiert die Auswahl für eine notwendigerweise stichprobenhafte Tiefenbohrung. Der Suchbegriff health dient dazu, sich der Gesamtzahl der Artikel anzunähern, um die Häufigkeiten der anderen Begriffe in Verhältnis stellen zu können.

In der Gesamtsicht der drei Zeitschriften fällt erstens die ansteigende Häufigkeit von Begriffen aus dem Wortfeld der Wissenschaft auf. Merkliche Anstiege in der Abundanz finden sich bei research, survey, questionnaire und Evaluat*. Dabei indizieren die Begriffe eine Tendenz zu sozialwissenschaftlichen Methoden der Wissensgewinnung, speziell der Umfragefor- 
schung, in Form von Meinungs- und Einstellungsforschung. Diese scheinen ebenfalls die Beurteilungskriterien für den in seiner Häufigkeit (insbesondere in den Health Education Monographs und dem International Journal of Health Education) rapide ansteigenden Bewertungs- und Begutachtungsprozess von Projekten zu operationalisieren (Evaluation).

Die Begriffe stammen zweitens aus dem semantischen Feld des Verhaltens, der Einstellung und Gewohnheit, der Überzeugung und der Motivierung/Motivation. Zusammen mit dem durchgehend häufig verwendeten Begriff des Wissens „boomten“ in den 1960er Jahren auch diejenigen aus dem Umfeld von Verhalten und Verhaltensänderungen (Überzeugung/ Überzeugen, Gewohnheit oder Motivation). Allen voran scheint Haltung/ Einstellung gerade in den neuen Zeitschriften, den Health Education Monographs und dem International Journal of Health Education, ein wichtiges Thema der zweiten Hälfte der 1960er Jahre gewesen zu sein. Es liegt also nahe, für die weitere qualitative Auswertung auf Artikel aus allen drei Zeitschriften genauer einzugehen, in welchen sich die Begriffe mit Konjunktur verdichteten.

Tab. 1 Begriffshäufigkeiten ${ }^{a}$

\begin{tabular}{|c|c|c|c|}
\hline \multirow[t]{2}{*}{ Suchphrase } & $\begin{array}{l}\text { Trefferanzahl } \\
\text { The Health } \\
\text { Education } \\
\text { Journal (GB) }\end{array}$ & $\begin{array}{l}\text { Trefferanzahl } \\
\text { Health Education } \\
\text { Monographs (USA) }\end{array}$ & $\begin{array}{l}\text { Trefferanzahl } \\
\text { International } \\
\text { Journal of Health } \\
\text { Education (F/CH) }\end{array}$ \\
\hline & 1943-1969 & 1957-1969 & 1958-1969 \\
\hline Health & 227 & 133 & 326 \\
\hline 1943-1949 & 38 & - & - \\
\hline 1950-1959 & 83 & 15 & 21 \\
\hline 1960-1964 & 54 & 34 & 135 \\
\hline 1965-1969 & 52 & 82 & 170 \\
\hline$=>1960-1969$ & 106 & 118 & 305 \\
\hline Behav* & 96 & 127 & 112 \\
\hline 1943-1949 & 11 & - & - \\
\hline 1950-1959 & 36 & 13 & 1 \\
\hline 1960-1964 & 20 & 29 & 38 \\
\hline 1965-1969 & 29 & 85 & 73 \\
\hline$=>1960-1969$ & 49 & 114 & 111 \\
\hline Persua* & 55 & 37 & 11 \\
\hline 1943-1949 & 11 & - & - \\
\hline 1950-1959 & 14 & 6 & - \\
\hline
\end{tabular}


Tab. 1 (Fortsetzung)

\begin{tabular}{|c|c|c|c|}
\hline \multirow[t]{2}{*}{ Suchphrase } & $\begin{array}{l}\text { Trefferanzahl } \\
\text { The Health } \\
\text { Education } \\
\text { Journal (GB) }\end{array}$ & $\begin{array}{l}\text { Trefferanzahl } \\
\text { Health Education } \\
\text { Monographs (USA) }\end{array}$ & $\begin{array}{l}\text { Trefferanzahl } \\
\text { International } \\
\text { Journal of Health } \\
\text { Education (F/CH) }\end{array}$ \\
\hline & 1943-1969 & 1957-1969 & 1958-1969 \\
\hline 1960-1964 & 11 & 9 & 2 \\
\hline 1965-1969 & 19 & 22 & 9 \\
\hline$=>1960-1969$ & 30 & 31 & 11 \\
\hline Habit* & 106 & 43 & 38 \\
\hline 1943-1949 & 18 & - & - \\
\hline 1950-1959 & 36 & 3 & - \\
\hline 1960-1964 & 25 & 7 & 16 \\
\hline 1965-1969 & 27 & 33 & 22 \\
\hline$\Rightarrow>1960-1969$ & 52 & 40 & 38 \\
\hline Motivat* & 42 & 78 & 55 \\
\hline 1943-1949 & 3 & - & - \\
\hline 1950-1959 & 16 & 11 & - \\
\hline 1960-1964 & 7 & 26 & 18 \\
\hline 1965-1969 & 16 & 41 & 37 \\
\hline$\Rightarrow>1960-1969$ & 23 & 67 & 55 \\
\hline Attitude & 121 & 98 & 83 \\
\hline 1943-1949 & 22 & - & - \\
\hline 1950-1959 & 46 & 12 & 1 \\
\hline 1960-1964 & 23 & 23 & 33 \\
\hline 1965-1969 & 30 & 63 & 49 \\
\hline$=>1960-1969$ & 53 & 86 & 82 \\
\hline Research & 101 & 115 & 133 \\
\hline 1943-1949 & 13 & - & - \\
\hline 1950-1959 & 43 & 13 & 1 \\
\hline 1960-1964 & 27 & 23 & 51 \\
\hline 1965-1969 & 18 & 79 & 81 \\
\hline$=>1960-1969$ & 45 & 102 & 132 \\
\hline Survey & 82 & 66 & 41 \\
\hline 1943-1949 & 9 & - & - \\
\hline 1950-1959 & 28 & 7 & 2 \\
\hline 1960-1964 & 16 & 12 & 22 \\
\hline 1965-1969 & 29 & 47 & 17 \\
\hline$\Rightarrow>1960-1969$ & 45 & 59 & 39 \\
\hline Questionnaire & 38 & 42 & 15 \\
\hline 1943-1949 & - & - & - \\
\hline
\end{tabular}


Tab. 1 (Fortsetzung)

\begin{tabular}{|c|c|c|c|}
\hline \multirow[t]{2}{*}{ Suchphrase } & $\begin{array}{l}\text { Trefferanzahl } \\
\text { The Health } \\
\text { Education } \\
\text { Journal (GB) }\end{array}$ & $\begin{array}{l}\text { Trefferanzahl } \\
\text { Health Education } \\
\text { Monographs (USA) }\end{array}$ & $\begin{array}{l}\text { Trefferanzahl } \\
\text { International } \\
\text { Journal of Health } \\
\text { Education }(\mathrm{F} / \mathrm{CH})\end{array}$ \\
\hline & 1943-1969 & 1957-1969 & 1958-1969 \\
\hline 1950-1959 & 11 & 1 & - \\
\hline 1960-1964 & 13 & 7 & 9 \\
\hline 1965-1969 & 14 & 34 & 6 \\
\hline$=>1960-1969$ & 27 & 41 & 15 \\
\hline Evaluat* & 62 & 96 & 85 \\
\hline 1943-1949 & 4 & - & - \\
\hline 1950-1959 & 21 & 7 & - \\
\hline 1960-1964 & 18 & 22 & 28 \\
\hline 1965-1969 & 19 & 67 & 57 \\
\hline$=>1960-1969$ & 37 & 89 & 85 \\
\hline Exhibit* & 70 & 23 & 18 \\
\hline 1943-1949 & 14 & - & - \\
\hline 1950-1959 & 35 & 4 & - \\
\hline 1960-1964 & 9 & 9 & 10 \\
\hline 1965-1969 & 12 & 10 & 8 \\
\hline$=>1960-1969$ & 21 & 19 & 18 \\
\hline Museum & 23 & $2^{b}$ & 6 \\
\hline 1943-1949 & 5 & - & - \\
\hline 1950-1959 & 12 & 1 & $2^{c}$ \\
\hline 1960-1964 & 4 & - & $1^{d}$ \\
\hline 1965-1969 & 2 & 1 & $3^{e}$ \\
\hline$=>1960-1969$ & 6 & 1 & 4 \\
\hline
\end{tabular}

${ }^{a}$ Fett hervorgehoben sind die je zweithäufigsten Trefferzahlen pro Zeitschrift sowie Veränderungen um das Dreifache bei Trefferzahlen größer als eins.

bIn beiden Fällen findet sich der Begriff jeweils in der Bibliografie von Artikeln, 1959 in „Papers of Peabody Museum of American Archeology and Ethnology“, 1967 in „Atomic Energy Museum".

${ }^{C}$ Entspricht einer Anzeige des Kölner Gesundheits-Museums im International Journal of Health Education 1959 sowie der Ankündigung eines Seminars des Bundesausschusses für gesundheitliche Volksbelehrung mit Besuch des DGM 1958.

${ }^{d}$ Verweis auf ein medical museum in Philadelphia des 18. Jahrhundert: 1961: 4.

" "Visitor reaction study undertaken in a US health museum" (1967: 10) sowie die Ankündigung eines Seminars am ehemaligen Kölner Gesundheits-Museum (vgl. 1969: 43). 


\section{Die Ver(sozial)wissenschaftlichung der Gesundheitsaufklärung im Modus der Gesundheitserziehung - qualitativ}

Eine erste Auswahl fällt auf Clair Elsmere Turners (1890-1974) Artikel „World Progress and Problems in Health Education“ 1964 im International Journal of Health Education, in dem unter der Fragestellung „Where, exactly, do we stand now?" alle relevanten Schlagworte zu finden sind. In der USamerikanischen fachimmanenten Erinnerungsarbeit der öffentlichen Gesundheit/der Gesundheitswissenschaften werden Turner das Thema und das Design der Malden-Studien aus den 1920er Jahren zugeschrieben (vgl. Sorensen 1987). Diese ersten mit Studien- und Kontrollgruppen arbeitenden Interventionsstudien der Gesundheitserziehung hatten kindliche Wachstumsraten mit schulischer Gesundheitserziehung positiv korreliert (vgl. Turner 1925, 1928). Die Untersuchungen stabilisierten zudem eine disziplinäre Perspektive der health education im Kern der Professionalisierungsbemühungen einer beruflichen Tätigkeit, die die Wissensvermittlung explizit dem Zweck der Handlungsänderung instrumentell unterordnete. Bereits 1926 hatte Turner, Professor für Biologie und Public Health am Massachusetts Institute of Technology in seinem Handbuch Personal and Community Health programmatisch formuliert: „Your health depends, not upon what you know, but upon what you do." (Turner 1926: 1; Turner 1952: 18) 1937 führte er weiter aus: „Knowledge alone, however, will not improve your health [...]. Good health is a state of complete physical efficiency. It is developed by habit, by custom, by doing, and not by merely thinking, or wishing, or knowing" (Turner 1937: 23). Auch in der neunten Ausgabe seines Handbuchs, das bis zu Turners Tod 1974 in insgesamt 14 Auflagen erschien, hielt er an dieser Prämisse metaphernfreudig fest: „Better health will come, of course, not from the mere acquisition of health knowledge, but from its application [...] Obviously health knowledge will not produce health until it is used. Fuel will not produce heat until it is burned, but that does not render it useless." (Turner 1952: 18). ${ }^{24}$ In den 1950er und 1960er Jahren fungierte Turner in internationalen Organisationen wie der WHO und der Internationalen Union für Gesundheitserziehung als einflussreicher Berater. In dieser Zeit war der Nestor der US-Gesundheitserziehung Chief Officer of Health Education der WHO, High Counsellor der IUHEP sowie Chief Advisor des International Journal of Health Education tätig, wo seine Stimme besonderes Gewicht hatte. Zudem übernahm er Anfang der 1960er Jahre die Beratung eines gemeinsamen WHO- und UNESCOProjekts zur Gesundheitserziehung (vgl. Viborel 1958a).

1964 zog Turner in einem Originalbeitrag in dieser Zeitschrift zur Gesundheitserziehung in Schulen Bilanz und wagte es, in die Zukunft zu blicken: health education sei im Kern damit beschäftigt, den Spalt zwi- 
schen Gesundheitswissen und Gesundheitshandeln zu überbrücken. Einer solchen Aufgabe könne eine Erziehungspraxis nur mithilfe von Wissen aus den psycho-social sciences nachkommen: „So health education uses knowledge from the fields of psychology, sociology, cultural anthropology and economics to influence the health knowledge, attitudes and behaviour of people“ (Turner 1964: 107). So wie Wissensbestände aus diesen Bereichen die Brücke zwischen Wissen und Handeln bauen helfen sollten, so beschrieb Turner auch, dass sich auf dieser Brücke Einstellungen/Haltungen (attitudes) befinden müssten. Einstellungen, Wissen und Verhalten - den Zusammenhang zwischen diesen drei Faktoren des Gesundheitshandelns sollten die Referenzwissenschaften aufklären.

Was die Gesundheitserziehung nach Turners Auffassung komplex machte, war die Überzeugung, dass Handel schwierig zu verstehen und noch diffiziler zu ändern war. So viele Faktoren beeinflussten individuelles Verhalten:

To change behaviour is not always easy. The causes of human behaviour lie in the purposes, beliefs, convictions and understandings of people - the ways in which they perceive themselves and the world in which they live. The same situation produces different behaviour in different individuals (Turner 1964: 108).

Aus der Erkenntnis der sozial situativen und psychologisch individuellen Bestimmung des Verhaltens folgerte Turner, dass Gesundheitserziehung nicht funktionieren könne, ohne Informationen über die Selbstverhältnisse von Individuen zu ihrer sozialen Welt zu sammeln. Ohne Wissen über gesundheitsrelevante Überzeugungen und Verständnisse blieben auch einer organisierten, kooperativen und koordinierten Gesundheitserziehung jegliche Chancen auf Erfolg verwehrt. Ein health educator müsse deshalb die Sozial- und Machtstruktur, die Überzeugungen, Traditionen, Sitten, Vorurteile und jeweiligen Gemeindeorganisationen im Vorhinein genau untersuchen, bevor eine Aufklärungskampagne gestartet werde. Das könne jedoch noch gar nicht Forschung in der Gesundheitserziehung genannt werden, sondern markiere lediglich professionelles Handeln, so Turner. Gesundheitserzieherische Forschung selbst könne sich nur auf die Beobachtung und Beurteilung der Mittel beschränken, mit denen Verhaltensweisen zu ändern seien: „It is the study of educational methods which builds skills in developing desireable knowledge, attitudes and behaviour" (Turner 1964: 113; vgl. ebenso: Dalton et al. 2007: 2-67). Als Methode favorisierte Turner dabei ein evaluatives Vorgehen: Die Qualitätsbeurteilung von Programmen der Gesundheitserziehung könne nur aus ihren Effekten heraus vorgenommen werden: "good programmes produce results“ (Turner 1964: 108). Dafür wiederum brauchte es eine Zieldefinition im Vorfeld, 
an der die Wirksamkeit der health education Maßnahmen sowohl laufend als auch im Nachhinein gemessen werden konnte.

Die Ver(sozial)wissenschaftlichung des Verhaltens, die das eigene professionelle Vorgehen wie auch die Gesundheitserziehungsforschung nunmehr prägen sollte, implizierte für die Gesundheitserzieher*innen eine Flexibilisierung der Normbestimmung von Gesundheit und deren Durchsetzung (vgl. Link 1997). Gesunde Handlungsweisen festzulegen und zu erreichen durfte nicht alleine aus dem Wissen der Expert"innen heraus begründet werden: „Health education does not seek to control the behaviour of groups against their wills" (Turner 1964: 110). Jegliche Gesundheitserziehung müsse am Ende den Einzelnen die Wahl lassen, das Wissen anzunehmen, Einstellungen zu modifizieren und Verhaltensweisen zu ändern. Menschen mussten überzeugt werden. Sie mussten dafür gewonnen werden, sich ein gesundheitsförderliches Wissen und Einstellungen anzueignen, die entsprechendes Handeln nach sich ziehen sollten. Das machte Gesundheitserziehung in Turners Augen zu einer Praxis der Öffentlichkeitsarbeit:

The development of such knowledge and favourable attitudes, whether through mass media or through the contact of the public with public health personnel, is public relations (Turner 1964: 110).

Die Vorstellung einer modernen health education als „applied behavioural science" barg das Potenzial, die Normen von Gesundheit und Gesundheitserziehung zu subjektivieren (Turner 1964: 114). Denn zusammen mit der Wertsetzung, dass die Entscheidung über die Annahme der Gesundheitserziehungsangebote beim Individuum liege, wertete die sozialwissenschaftliche Repräsentierung des Wissens, der Einstellungen und der Handlungsweisen die Eigensinnigkeit der avisierten Zielkollektive auf. Das Ideal des mündigen Bürgers schien hier durch: Es verlagerte die Verantwortlichkeit für Gesundheit auf das Individuum. Aber es machte auch andere subjektive Prioritäten sichtbar und sprach diesen Geltung zu - gewissermaßen als eine Art Vetorecht gegen die Gesundheitserziehung. ${ }^{25}$

Genau diese potenzielle Aufweichung der Grenze zwischen Devianz und Norm vollzogen Stimmen aus den sozialistischen Ländern nicht. Nur ein Jahr nachdem Rolf Thränhardt Forschung auf dem Gebiet der Gesundheitserziehung in der DDR als noch junge Unternehmung bezeichnet hatte, in der wenig Erfahrung vorliege, präsentierte sich Čenĕk Adamec aus dem Zentralinstitut für Gesundheitserziehung in Prag elaborierter. Auf Grundlage der ebenfalls expliziten Trennung von knowledge, attitude und behavior skizzierte er 1960 den Stand der Gesundheitserziehung in der ČSSR. Eine erste Voraussetzung der Gesundheitserziehung bestehe darin, qualitative Vorerhebungen des Istzustandes von Gesundheitswissen, -ein- 
stellungen und -handeln mithilfe von Methoden der Umfrageforschung durchzuführen. Nur auf dieser Basis könnten die Ziele einer jeweils zu entwerfenden Kampagne der Gesundheitserziehung bestimmt und ihr Erfolg ex post abgeschätzt werden. Die Erfahrung, so Adamec, lehre, dass es mit reiner Informationsvermittlung nicht mehr getan sei: „In some instances there is no point in continuing to propagate certain facts [...] and it is necessary to seek means of influencing the behaviour of the population" (Adamec 1960c: 83). Auch hier wurde eine qualitative Umfrageforschung zu Gesundheitswissen und Gesundheitseinstellungen zum Ausgangspunkt einer Gesundheitserziehung erhoben, die vorrangig auf die Veränderung von Verhaltensweisen abzielte. Denn ohne über Denkmuster („ways of thinking“) und Handlungsmotivationen („motivation of their behaviour“) informiert zu sein, tappe die Gesundheitserziehung nur im Dunkeln („not knowing whom he is addressing, not knowing the response of his audience“; Adamec 1960c: 84).

Adamec mobilisierte auch die qualitative Sozialforschung zur Mittelauswahl und zur Wirksamkeitsbeurteilung der Gesundheitserziehung. Seine Artikel in allen drei untersuchten Zeitschriften, die dort zudem als Referenzen in anderen Artikeln genannt wurden, behandelten zumeist den Nutzen der Umfrageforschung für die Gesundheitserziehung, ihre Bedingungen sowie ihre medialen Formen. ${ }^{26}$ Indem Adamec die Gesundheitserziehung als Kommunikation verstand, deren Aufgaben, Inhalte und Formen vom Zielpublikum aus zu bestimmen und $\mathrm{zu}$ beurteilen seien, übernahm er einen Antrieb zur Flexibilisierung der gesundheitserzieherischen Normen. Doch Adamec machte sich nicht die Leitfigur des mündigen Bürgers zu eigen. Er entkoppelte die Mittelbestimmung und Qualitätsbeurteilung der Gesundheitserziehung von der Notwendigkeit der Überzeugung zu einer freiwilligen Rezeption der Gesundheitserziehungsangebote. Das funktionierte über den Begriff des Bewusstseins: Vorbereitende Forschung zum Zustand des Gesundheitswissens, der -einstellungen sowie zum -verhalten wurde damit gleichgesetzt, Lücken im Gesundheitsbewusstsein („gaps in the health consciousness“; Adamec 1960c: 84) zu detektieren (vgl. ebenso Adamec 1962; Adamec 1961). Widersprüche zwischen Ist- und Sollzustand wurden als Bewusstseinsmangel definiert und somit vom Gesundheitsideal der Gesundheitserzieher aus gedacht. Der Unterschied zwischen Turner und Adamec bestand also in den divergenten Subjektleitbildern und dadurch im Grad, die Mittel, Formen und Ziele der Gesundheitserziehung vom avisierten Zielpublikum aus zu denken.

Gleichwohl stimmten Turner und Adamec in den gemeinsamen Tenor des internationalen Professionsnetzwerks der Gesundheitserzieher*innen ein, der in den Zeitschriften vorherrschte, und in dem vor allem Akteure aus den Vereinigten Staaten eine Publikationsführerschaft innehatten. 
Modernität bestand darin aus der evaluativen Ziel-, Mittel- und Wirksamkeitsbestimmung, aus dem prinzipiellen Abzielen auf eine Veränderung von Verhaltensweisen und aus einem komplexen Verständnis von Verhalten und Kommunikation. Genau diese dreifache Verschiebung bezeichnete 1961 unter anderem der Mediziner, Medizinhistoriker, Gesundheitswissenschaftler und Professor für Public Health Education an der Columbia University, George Rosen (1910-1977), als jüngste Trends in der Gesundheitserziehung sowie als Motor einer (weiteren) Akademisierung des Berufsfeldes der Gesundheitserzieher*innen. Health education sei im Wesentlichen ein sozialer Prozess, der auf die Intervention in soziale Prozesse abziele: „Health education is complex, as much a social product as the people and agencies it is intended to affect" (Rosen 1961: 505). Mit dieser Perspektive müssten sich die Gesundheitserzieher*innen den Sozialwissenschaften zuwenden - nicht nur, um die eigenen Aktivitäten zu beurteilen, sondern auch, um überhaupt zu verstehen, wie sie mit Individuen oder Gruppen kommunizieren könnten. Konsequenterweise müsse der althergebrachte "rationalistische“ Ansatz fallen gelassen werden. Um Wirkung zu entfalten, dürften die „Fakten“ nicht einfach „heruntergereicht“ werden. Die Annahme, dass Menschen die Wahrheit anerkennen, wenn ihnen die Sachverhalte präsentiert würden, und daraus auch noch die notwendigen Schlussfolgerungen für ihr Handeln zögen, habe sich als irrig herausgestellt (vgl. Rosen 1961: 504f.).

Wenn ein rationalistischer Ansatz für die Gesundheitserziehung nicht mehr infrage kam, was konnte dann Gesundheitshandeln beeinflussen und was konnten die Sozialwissenschaften dazu beitragen? Das Problemfeld der vielfältigsten Determinationen individuellen Verhaltens war bereits 1959 auf der Jahrestagung der American Public Health Association prominent von deren Health Education Section abgesteckt worden. Der Sozialpsychologe Eugene Hartley (1912-2002) widmete sich den psychologischen Bedingungen des individuellen Verhaltens, der Medizinsoziologe Robert Straus den soziologischen und Margaret Mead (1901-1978) den kulturellen Konditionen. Alle drei betonten nachdrücklich, dass individuelles Handeln in Interaktion mit der Umwelt erfolge und sich vor allem durch Variabilität auszeichne. Sie plädierten daher für eine holistische Verhaltenstheorie („prejudice in favor of an integrated or holistic theory of behavior“; Straus 1961: 1548). Angesichts der Komplexität der Einflüsse auf menschliches Handeln erschien es den Autor*innen bestenfalls möglich, Determinanten aus einer analytischen Ordnung der vielfältigen Interaktion - quasi Idealtypen - benennen zu können (vgl. Hartley 1961: 1546). Für Hartley waren individuelle Einstellungen und Überzeugungen Gruppenprodukte. Daher bestehe der beste Forschungsansatz darin, sich mit dem Konzept der sozialen Rollen den jeweiligen Gruppen zuzuwenden, in denen Ver- 
haltensweisen, Werte, Einstellungen und Überzeugungen bestärkt würden. Anschließend sollten die psychologischen Prozesse des Einzelnen innerhalb seines sozialen Settings betrachtet werden (vgl. Hartley 1961: 1543). ${ }^{27}$ Straus machte sich darüber hinaus stark für den Respekt gegenüber der Diversität von sozialen Sinnstiftungen und Handlungsweisen in Bezug auf Krankheitserfahrungen. Das Verständnis der subjektiven Erfahrung von Krankheit und Gesundheit, die nur in Wechselwirkung mit der gesellschaftlichen Umwelt zu denken sei, habe die Arbeit der Gesundheitserziehung zu orientieren (vgl. Straus 1961: 1551). Noch expliziter setzte sich Margaret Mead für die Strukturierung und Beurteilung der Gesundheitserziehung nach den Vorstellungen ihrer Zielgruppen ein. Nicht nur müsste zuerst eine kulturelle Kommunikationsfähigkeit zwischen Erzieher und zu Erziehenden hergestellt sein. Der Erzieher sei darüber hinaus auch moralisch verpflichtet, die Ganzheit (wholeness) der Menschen zu respektieren. Gesundheitserzieher*innen sollten die Kapazität zum Genießen von Freude, Risiko und sozialen Bindungen fördern, ohne Angst oder Bedrohung als Strategien der Ansprache zu nutzen oder Eigeninteressen zu folgen. Im Kern verstand Mead damit Soziabilität und Empfindungsfähigkeit als Gesundheit (vgl. Mead 1961: 1554). Die Autor*innen komplizierten moralisch unterbaut das individuelle Verhalten und die Möglichkeiten seiner Beeinflussung. Sie wiesen der Gesundheitserziehung aber auch eine modern erscheinende Option zu, diese Vielschichtigkeit bearbeiten zu können - durch mehr Einstellungs-, Wirkungs- und Kommunikationsforschung.

$\mathrm{Zu}$ den empfohlenen Inspirationsquellen einer health education-Forschung gehörten die soziologischen Konzepte der relativen Deprivation, des Rollensets und der sozialen Referenzgruppe, die von Straus und Hartley rezipiert worden waren (vgl. Blackwell 1964) ${ }^{28}$ Kurt Lewins (1890-1947) Forschungen zur Gruppendynamik und die an ihn anschließenden Arbeiten (vgl. Nyswander 1961) wurden in den Zeitschriften ebenfalls mehrfach erwähnt. Aber auch Leon Festingers (1919-1989) Theorie der kognitiven Dissonanz wurde dankbar aufgenommen, da sie die Diskrepanz zwischen Wissen, Meinungen, Überzeugungen, Einstellungen und Verhaltensweisen als Quelle von Leid und damit als Antrieb zur Verhaltensänderung interpretierte. Gleichwohl räumte Festinger ein, dass die Auflösung von Qual nur eine Kognitions- und Handlungsmotivation unter vielen sei und darüber hinaus Dissonanz sich fast immer dann einstellen würde, wenn man durch Belohnung oder Strafe ein bestimmtes Verhalten evozieren wolle. Erfolgversprechender sei daher, eine soziale Wirklichkeit herzustellen, welche die erwünschte Kognition bestärke. Auch Festinger stellte damit Verhalten und Kognition in einen interaktionalen Zusammenhang mit der sozialen Umwelt. Er richtete den Blick der Gesundheitserzieher*innen auf positive oder negative soziale Images von avisierten Verhaltensweisen und 
auf den Einfluss der jeweiligen Referenzgruppen auf diese. Diese Perspektivierung hatte Festinger sogar selbst vobereitet: Genau der Widerspruch, der die Gesundheitserziehung langanhaltend beschäftigen sollte - der zwischen dem Wissen um die Gesundheitsschädlichkeit des Rauchens und der ungebrochenen Rauchinzidenz -, hatte ihm 1957 als Illustrationsbeispiel seiner Theorie gedient (vgl. Festinger 1978 [1957]: 15-35, 253-273; Zander 1962).

Auch die Vorstellung vom Funktionieren von (massenmedialer) Kommunikation wurde unter dem Einfluss der behavioural sciences vielschichtiger. Von Paul Lazarsfeld (1901-1976) und Elihu Katz entliehen die Gesundheitserzieher*innen die Erkenntnis, dass massenmediale Inhalte nur selektiv rezipiert oder missverstanden wurden (vgl. Kegeles 1963; Ewbank 1966). Doch Katz' und Lazarsfelds Arbeiten speisten wie auch Festingers Theorie die Hoffnung auf Wirksamkeit. Da massenmediale Information in zwei Schritten fließe, von den Medien zu „Meinungsführern“ und von diesen zum eigentlichen Zielpublikum, mussten die opinion leaders als Multiplikatoren erreicht werden. In lokal situierten Kontexten mussten diese Informationen zertifizieren, Vertrauenswürdigkeit belegen und auf diese Weise Einstellungen und Vorstellungen prägen (vgl. Griffiths 1957; Antonovsky \& Shuvai 1966; Krister 1967).

Die Verhaltenswissenschaften machten den Gegenstand der Gesundheitserzieher*innen insgesamt komplexer: Verhalten, Kommunikation und Gesundheit wurden als sozial bestimmt betrachtet (vgl. Straus 1961: $1547 \mathrm{f}$.). Die Erkenntnis, dass gesundheitsrelevante Einstellungen und Überzeugungen divers, mitunter widersprüchlich und schwierig zu verändern seien, verfestigten auch Mayhew Derryberry (1902-1979), sein Nachfolger Godfrey Hochbaum (1916-1999) sowie dessen Mitarbeiter Irwin M. Rosenstock (1925-2001). ${ }^{29} 1941$ hatte Derryberry die Leitung der Abteilung Health Education des United States Public Health Service übernommen und sich der sozialpsychologischen Forschung zum Gesundheitsverhalten gewidmet. Aus der Suche nach Erklärungen für das Scheitern von nationalen Programmen der Tuberkulosereihenuntersuchung entstand dort das Health-Belief-Modell (das Modell gesundheitlicher Überzeugungen). Dieses hatte zwischen Angebot und Nachfrage von krankheitspräventiven Maßnahmen ein Zusammenspiel von Überzeugungen und Wahrnehmungen eingeführt: Die Rezeption von Präventionsmaßnahmen sei abhängig von der individuellen Einschätzung der jeweils eigenen Risikolage, der Schwere möglicher gesundheitlicher Beeinträchtigungen sowie von dem Ergebnis der Verrechnung von eingeschätzten Vor- und Nachteilen einer Verhaltensänderung. Außerdem besäßen Handlungsratschläge nur Chancen auf Adaption, wenn diese auch im Alltag praktikabel seien (vgl. Glanz \& Bishop 2010: 402). Wie Turner fungierte auch Derryberry 
als Elder Statesman einer Ver(sozial)wissenschaftlichung der Gesundheitsaufklärung im Modus der Gesundheitserziehung. Er stand 1960 dem Forschungskomitee der IUHEP vor, als Turner die Rolle des Chief Advisor der Unionszeitschrift (International Journal of Health Education) ausfüllte (vgl. Derryberry 1960b).

Derryberry, Hochbaum und Rosenstock wiesen einen Weg durch die Netze multifaktorieller Wechselwirkungen, in die das menschliche (Gesundheits-)Handeln durch seine sozialwissenschaftliche Perspektivierung geraten war: mehr Wissenschaft in Form einer evaluativ verfahrenden Forschung (vgl. Hochbaum 1965: 148). Genau dies setzte Derryberry 1960 den Gesundheitserzieher*innen auf die Agenda (vgl. Allegrante et al. 2004). Aus der bisherigen Forschung müsse sich weitere Forschung ergeben - eben auch zu den sozialen und kulturellen Charakteristika der Menschen.

Research in health education then is concerned with the process by which people change their health behaviour. It includes study of all the various factors in the process and the dynamics of the relationship between these factors. For example, health education research encompasses studies of the social and cultural characteristics of the people, the character of the health practice being advocated and the educational methods and materials used in bringing about the change or improvement in health behaviour (Derryberry 1960b: 164).

In diesem Zuschnitt war Gesundheitserziehung in der Tat eine angewandte Verhaltens- und Kommunikationsforschung geworden. Derryberry definierte sie im selben Jahr folgendermaßen:

Health education is concerned with effecting improved health attitudes and behavior in the general public. Accomplishment of this goal requires careful and thorough consideration of the present knowledge, attitudes, goals, perceptions, social status, power structure, cultural traditions and other aspects of whatever public is to be reached. Only in terms of these elements can a successful program be built (Derryberry 1960a: 9).

Das sich in den Zeitschriften diskursiv gebildete Professionsnetzwerk der Gesundheitserzieher*innen bemühte sich darum, ein international verbreitetes Wissenskorpus zu schaffen, an dem die Expert*innen ihre Prinzipien und Praxis ausrichteten sowie ihren Status als professionals banden. Doch bereits in den 1960er Jahren war ihr Wissen - durch die konsequente Steigerungslogik der Ver(sozial)wissenschaftlichung - so komplex und unübersichtlich geworden, dass es einer sytematisierenden Ordnung bedurfte. 1962 und 1963 sowie 1967 bis 1969 erschienen in den Health Edu- 
cation Monographs unter Turners Mitarbeit ausführliche Literaturberichte. In den Überblicksdarstellungen wurden bisherige Studienergebnisse sowie die verteilt publizierten Erfahrungen gesammelt, gesichtet und ausgewertet (Young et al. 1963; Young et al. 1967-1969). Das Schrifttum wurde in die Rubriken Gesundheitswissen, Gesundheitsvorstellungen, Gesundheitshandeln (1), psychosoziale und kulturelle Einflussfaktoren (2), Methoden und Materialien der Gesundheitserziehung (3), Programmplanung und -evaluation (4) aufgeteilt. 1967 kamen noch patient education und school health education als situative Kategorien der Stoffgliederung hinzu.

\section{Zusammenfassung und Ausblick: Die Ver(sozial)wissenschaftlichung der Gesundheitsaufklärung und ihre Grenzen}

In den 1950er Jahren entwickelte sich ein anglofoner, internationaler Fachdiskurs der Gesundheitserzieher*innen, der sich in den 1960er Jahren in mehreren Zeitschriften etablierte. Darin tauschten Akteure sich über ihre Strukturen, Forschungen und Erfahrungen aus und konstituierten damit einen Kommunikationsraum, in dem sich ein esoterisches Wissenskorpus herausbildete. Auf diesem Parkett manifestierte sich eine Art Denkkollektiv, das durch das gemeinsame Gestaltsehen verbunden war, nach den besten Mitteln zur gesundheitlich positiven Beeinflussbarkeit individuellen Handelns zu suchen und die Antworten mit Methoden der Umfrage- und Evaluationsforschung zu finden. Ein solcher nun "Gesundheitserziehung“ genannter Modus der Gesundheitsaufklärung hatte seinen Gegenstand und sein Instrumentarium mit Methoden der empirischen Sozialforschung beständig zu beobachten, zu evaluieren und nachzujustieren. Als kommunikativ angewandte Verhaltenswissenschaft nutzte sie zielgruppenspezifisch Massenmedien wie Presse, Rundfunk, Film und Fernsehen, denen gesundheitserzieherische Effektivität und Effizienz zugeschrieben wurde. Ausstellungen hingegen gehörten prioritär nicht mehr dazu. Die Grundlage dieser Perspektive bildete die wiederum aus den Sozial-, Verhaltens- und Kommunikationswissenschaften entlehnte Erkenntnis, dass soziales Handeln in Netzen wechselseitiger Abhängigkeitsfaktoren verfangen und die Möglichkeit seiner Beeinflussung schwierig war. Einstellungen und Motivationen sowie Konzepte der kognitiven Dissonanz, selektiver Informationsrezeption, bestärkender Referenzgruppen, sozialer Images und Rollen hatten das individuelle Verhalten komplex gemacht. Die Gesundheitserzieher*innen aus beiden deutschen Staaten machten sich diesen Gestaltwandel zu eigen und passten ihn an die Subjektleitbilder, die Praxis sowie das institutionelle 
Feld der Gesundheitsaufklärung in der DDR oder der Bundesrepublik an. In der Bonner Republik übernahm die BZgA in den 1970er Jahren sogar maßgeblich die Trägerschaft des internationalen Professionalisierungsdiskurses der Gesundheitserzieher*innen im deutschsprachigen Raum.

Aus der Konklusion, dass sich die Probleme - die erst durch die verhaltenswissenschaftliche Perspektivierung der Gesundheitsaufklärung sichtbar gemacht worden waren - nur durch weitere Forschung lösen lassen würden, folgte eine Professionalisierungsstrategie der Gesundheitserziehung. Im Zusammenklang mit der in beiden deutschen Staaten offensichtlich demonstrierten politischen Verantwortlichkeit für die Gesundheit der Bürger schufen Gesundheitserzieher*innen in BRD und DDR aus der Krise des „Dresdner Modells“ heraus gegen Ende der 1960er Jahre Organisationen, welche ihre Agenda institutionalisierten. Sie brachten dafür eine Rhetorik in Anschlag, in deren Kern Wissenschaftlichkeit und die zentrale Steuerung als moderne Lösungen für moderne Probleme standen. Diese Vorstellung einer zeitgemäßen Gesundheitsaufklärung orientierte sich in erster Linie an den USA und wurde befestigt durch internationale Organisationen wie die WHO und die IUHEP. Auch durch diese Internationalität fiel die damalige, zukunftszugewandte Gegenwartsimagination der Gesundheitserziehung als eine verwissenschaftlichte Sozialtechnologie in beiden machtpolitischen Blöcken des Kalten Kriegs auf einen fruchtbaren Boden des Wissenschafts- und Machbarkeitsoptimismus.

Die Verwissenschaftlichung des Gesundheitshandelns hatte den Wechsel der Gesundheitsaufklärung in den Modus der Gesundheitserziehung befördert. Diese Umstellung wiederum verstärkte eine empirisch-evaluative Beobachtung des Gegenstandes und der eigenen Praxis. Mit dieser Selbst- und Fremdbetrachtung, die die Verwissenschaftlichung mit sich brachte, brauchte und einforderte, wurden Feedbackschleifen im Modus der Gesundheitserziehung etabliert, die Erfahrungen aus der eigenen Praxis in die Entwicklung weiterer Kampagnen zurückfließen ließen. Gesundheitserziehung ermöglichte dadurch in den 1970er Jahren ein institutionelles Lernen, indem Gesundheitserzieher*innen experimentierten, wie sie gesundheitsförderliche und krankheitspräventive Handlungsweisen formen konnten, immer wieder scheiterten und darüber erfuhren, wie wenig sie eigentlich wussten. Als Präsident Wolfgang Fritsche 1971 eine der ersten Kampagnen der BZgA gegen das Rauchen bei Jugendlichen auf der Second World Conference on Smoking and Health in London vorstellte, brachte er die Mühen und Nöte der Versuchsphase der Gesundheitserziehung auf den Punkt:

Almost nothing certain was known about how we get people to change their behaviour [...]. The campaign we started did not win the victory 
we expected. When we looked into the question of why the appeals distributed by schools failed to work we found that young adolescent teenagers did not trust adults [...]. Nevertheless, we stayed with the target population, because we believed the fact of distrust in this age group was an encouragement to try to get young people to do something for themselves [...]. In consequence we had to forget everything that we had assumed, believed in or wished to do, and start probing into the teenage population to see the problem of smoking and health entirely from their angle [...]. Then we started the pilot project under the assumption that the image of the young juvenile smoker could be devalued [...]. This did not work and we had to throw out the whole campaign and start anew [...]. We found that smoking for our target population was not a health problem and that there was no single reason for smoking but a hole group of motives - at least eight (Fritsche 1972: 77 f.).

Wie der stellvertretende Leiter der BZgA einige Jahre später schrieb, erfüllte sich „die [...] Hoffnung [...], richtige Rezepte für die Lenkung der Menschen im Sinne einer effektiven gesundheitlichen Aufklärung zu finden“ jedoch nicht (Schnocks o. J. (ca. 1981): 17). Die Ver(sozial)wissenschaftlichung der Gesundheitsaufklärung machte die Komplexität menschlichen Verhaltens sichtbar und erhöhte diese bis an den Rand seiner Unbeeinflussbarkeit immer weiter. Erfolgreich war der Modus der Gesundheitserziehung allerdings in einer anderen Hinsicht: Mit ihm war das Potenzial angestiegen, Lebenswelten der Zielgruppen sichtbar zu machen und Krankheitsprävention von diesen, von ihren Subjekten, aus zu denken.

\section{Anmerkungen}

1 Anonym (Wolfgang Fritsche): Gedanken zur Umwandlung des Deutschen Gesundheits-Museums. Zentralinstitut für Gesundheitserziehung e. V. in eine Bundeszentrale für Gesundheitserziehung, 15.05.1965. In: BArch. B 310/114: Deutsches GesundheitsMuseum Köln. Korrespondenz mit Vereinsträgern, 1962-1969: unpag., hier: 1 und 4. Vgl. hierzu ebenso: Sammer 2015: $265 \mathrm{f}$.

2 Den Begriff Gesundheitsaufklärung verwende ich als Terminus technicus für eine Praxis der gesundheitsrelevanten Wissensvermittlung über Massenmedien in jeweils spezifizierten Öffentlichkeiten mit dem Zweck der Informierung oder der Verhaltensänderung. Die Quellenbegriffe Hygienische Volksbelehrung und Gesundheitserziehung (sowie mit Abstrichen Health Education) bezeichnen bestimmte Modi der Gesundheitsaufklärung.

3 Entschließung der Gesundheitsministerkonferenz der Bundesländer vom 09.10.1964. In: Hopf (1976: 37 f.). 
4 Vgl. Stiftung Deutsches Hygiene-Museum (2001); Laukötter (2010); Roeßiger \& Schwarz (2011); Nikolow (2015b); Osten et al. (2015); Winkler (2015); Laukötter (2018). Vgl. Aronowitz (1998: 111-144); Rothstein (2008); Madarász (2009); Timmermann (2010); Weisz (2014).

6 Vgl. Berridge (2007: 23-80); Berridge (2010); Berlivet (2005); Berlivet (2008); Sammer (2015).

7 Vgl. beispielsweise Arbeiten zur Geschichte gesellschaftlicher Ordnungsvorstellungen und sozio-politischer Prozesse des Ordnens (Etzemüller 2009; Reinecke 2010a; Reinecke \& Mergel 2012; Eghigian 2015), die der Selbstverhältnisse in der Gesellschaft (Maasen et al. 2011; Tändler \& Jensen 2012a; Tändler \& Jensen 2012b; Tändler 2016), der Human- und Sozialwissenschaften (Weischer 2004; Brückweh et al. 2012; Link 2018) und ihrer Verbindung zur Politik (Sala 2017). Programmatische Beiträge erhoben die Annäherung der Sozialgeschichte an die Geschichte der Wissenschaften zur Möglichkeit, die Zeitgeschichte mit dem Quellen- wie auch Analysebegriff „Wissensgesellschaft" neu zu bestimmen (Szöllösi-Janze 2004a; Szöllösi-Janze 2004b und dazu auch Reinecke 2010b sowie Ziemann 2012).

8 Vgl. ferner Linek (2016); Pfütsch (2017); Thießen (2017); Tümmers (2017).

9 Entsprechend einer Suche in der Zeitschriftendatenbank nach dem Stichwort Gesundheitserziehung, health education, éducation de la santé/éducation sanitaire, гигиеническое воспитание (gigieničeskoe vospitanie). Bei der Revue internationale d'éducation de la santé handelt es sich um die französischsprachige Ausgabe des International Journal of Health Education.

10 Das betrifft beispielsweise die "monatliche Zeitschriftenrundschau“ Gesundheitsfürsorge, Gesundheitserziehung, die zwischen 1959 und 1963 vom Westberliner Senator für Gesundheitswesen und anschließend bis 1979 durch die Berliner Medizinische Zentralbibliothek herausgegeben wurde. Andere Public Health Journale wie auch Illustrierte (beispielsweise Gesundes Volk. Volkstümliche Zeitschrift für Gesundheitspflege und Fragen der Sozialversicherung) sind aus der Analyse ebenfalls ausgeschlossen, weil sie entweder Gesundheitsaufklärung nicht als alleinigen Gegenstand behandelten oder, weil mit ihnen explizit kein (methodologischer und konzeptioneller) Beitrag zur Disziplinentwicklung geleistet werden sollte.

11 Seiring, Georg: Protokoll der Sitzung des Vorstands des Deutschen Hygiene-Museums am 29.01.1931. In: Hauptstaatsarchiv Dresden. 13686, Nr. 48: Deutsches Hygiene-Museum. Protokolle der Vorstandssitzungen, 1913-1935: unpag.

12 Vgl. ebenso Rolf Thränhardt: Bericht über die Reise der Delegation der Gesellschaft zur Verbreitung wissenschaftlicher Erkenntnisse in die Sowjetunion vom 10. bis 26.06.1955; Ders.: Reisebericht Prag, 17.9. bis 23.09.1956. In: Hauptstaatsarchiv Dresden. 13658, Rb/3 Bd. 1: Deutsches Hygiene-Museum: Reiseberichte, Ausland, BRD, 1954-1958: unpag.; Studienreise von Rolf Thränhardt und Otto Kunkel in die SU, 23.9. bis 05.10.1959. In: BArch. DQ 1/20542: Ministerium für Gesundheitswesen. Sekretariat des Stellvertretenden Ministers Prof. Dr. Friedeberger, 1959: unpag.

13 Vgl. Harmsen, Hans: Praktische Methoden der Gesundheitserziehung. Ergebnisse einer Arbeitstagung vom 11.02. bis 15.02.1957. In: BArch. B 142/397: Bundesministerium für Gesundheitswesen. Bundesausschuss für gesundheitliche Volksbelehrung. Allgemein, 1953-1958: 279.

14 Die Volltextsuche ergab im Health Education Journal für den (trunkierten) Suchbegriff exhibit* 70 Treffer zwischen 1943 und 1970 ohne erkennbare zeitliche Häufung der jeweiligen Artikel. Dasselbe gilt für die Health Education Monographs (23 Treffer) sowie das International Journal of Health Education (18 Treffer). Im Health Education Journal finden sich 23 Artikel, welche den Begriff „Museum“ inhaltsrelevant beinhalten. Nur sechs davon finden sich in den gesamten 1960er Jahren, der letzte 1966. In den Health Education Monographs lässt sich keine relevante Nennung erkennen. Das trifft bis auf eine Ausnahme - einen Artikel Bruno Gebhards - auch für das International Journal of Health Education zu. 
15 Thränhardt, ins Ministerium befördert, sollte 1962 vermerken: „Sie [die Mitarbeiter*innen, C.S.] kapitulieren vor der Tradition des Hauses, ohne diese schöpferisch weiterzuentwickeln.“ Thränhardt: Vermerk 13. März 1962. In: BArch. DQ 1/5225: Ministerium für Gesundheitswesen. Abteilung Wissenschaft und Ausbildung. Sektor Forschung, 1961-1963: unpag.

16 Vgl. Bruno Gebhard: Dresden Revisited, 11.07.1961. In: Dittrick Medical History Center: Nachlass Bruno Gebhard. B 17, 7.14b: Dresden Revisited 1961, 1961. In Gebhards Bewerbung seines Museums spiegelte sich somit die allgemeine Museums- und Ausstellungskrise der 1960er Jahre (vgl. Heesen \& Schulze 2015: 9-11; Keweloh 2016: 66).

17 Minister für Gesundheitswesen (Max Sefrin) 1967: 40.

18 Bundesminister für Gesundheitswesen (Käte Strobel) 1967: 374

19 Dieser Ausspruch ist sowohl im Jahresbericht des Bundesausschusses für gesundheitliche Volksbelehrung vom August 1956 dokumentiert (BArch. B 310/115: Bundeszentrale für gesundheitliche Aufklärung. Bundesausschuss für gesundheitliche Volksbelehrung, 1957-1960: unpag., hier: 6) als auch in der Dokumentation der 3. Vollversammlung des NKGE (Schubert, Gerda und Rolf Thränhardt: Senkung des Krankenstandes durch gesundheitliche Aufklärung und Erziehung im Betrieb. Vortrag für die 3. Vollversammlung des NKGE am 03.12.1963. In: BArch. DQ1/22446: Ministerium für Gesundheitswesen. Komitee für gesunde Lebensführung und Gesundheitserziehung: Protokolle, 1962-1964: unpag., hier: 4).

20 Vgl. Pott (2005: 341); Schnocks (o.J.) (ca. 1981) sowie eigene Berechnung nach Sichtung des Bestands der halbamtlichen Druckschriften der BZgA im Bundesarchiv Koblenz sowie im Printarchiv der BZgA.

21 Vgl. bspw. Schubert, Gerda und Rolf Thränhardt: Senkung des Krankenstandes durch gesundheitliche Aufklärung und Erziehung im Betrieb. Vortrag für die 3. Vollversammlung des NKGE am 03.12.1963. In: BArch. DQ 1/22446: Ministerium für Gesundheitswesen. Komitee für gesunde Lebensführung und Gesundheitserziehung: Protokolle, 1962-1964; Archiv BZgA. Schrank J, Fach 45. Arbeitsgemeinschaft Sozial- und Wirtschaftsforschung: Jugendliche und Rauchen, 1969.

22 Für den internationalen Austausch zeichnete zwischen 1967 und 1989 die Psychologin Rosmarie Wachsmuth (Erben) verantwortlich, die zur jungen Generation von Verhaltenswissenschaftlern an der BZgA gehörte. Vgl. das Interview mit ihr in: Ruckstuhl 2011: 113-119.

23 Vgl. zu Fritsche: Personalakte Wolfgang Fritsche laut E-Mail der WHO Records and Archives an den Verfasser, 15.08.2017.

24 Vgl. ebenfalls: http://www.nytimes.com/1974/11/28/archives/dr-clair-e-turner-healtheducator.html (03.12.2018); konzeptionell ähnlich: Turner 1952: 19-22.

25 Vgl. hierzu ebenfalls das starke Plädoyer Dorothy Nyswanders (1894-1998) für eine Gesundheitserziehung der „offenen Gesellschaft“, die (Verhaltens-)Vielfalt wertschätze und fördere: Nyswander 1967: insbesondere 11.

26 Vgl. Adamec (1960c); Adamec (1960a); Adamec (1960b); Adamec \& Kliment (1968); Adamec (1963); Adamec \& Satánek (1965).

27 Der Begriff der sozialen Rolle verweist auf Ralph Linton und George Herbert Mead, mit denen wiederum eine am Pragmatismus orientierte, interaktionistische, US-amerikanische Handlungssoziologie verbunden ist. Vgl. Münch (2002: 265-289).

28 Diese Instrumente rekurrieren auf Robert Merton (1910-2003). Vgl. Münch 2004: 15-40.

29 Vgl. Derryberry (1960a); Hochbaum (1960) (der Festinger, Katz, Lewin und Merton als Referenzen angibt); Rosenstock (1960); Rosenstock (1961); Hochbaum (1965). 


\section{Literatur}

Adamec, Čeněk 1960a. A Flannelgraph-Quiz. International Journal of Health Education (3:1): $39-42$.

Adamec, Čeněk 1960b. Nutrition Education over the Telephone. International Journal of Health Education (3:4): 200-202.

Adamec, Čeněk 1960c. Surveys of Knowledge, Habits and Attitudes: Their Role in Planning Health Education. Health Education Journal (18): 78-85.

Adamec, Čeněk 1961. Investigating Health Consciousness. International Journal of Health Education (4:3): 108-112.

Adamec, Čenĕk 1962. Pictorial Charts: Investigation of Health Consciousness. Health Education Journal (20): 105-108.

Adamec, Čenĕk 1963. Seasonal Problems in Nutrition. International Journal of Health Education (6): 21-23.

Adamec, Čeněk und Peter Kliment 1968. Educational TV Health Programmes: Some Survey Results. Health Education Journal (27:1): 12-16.

Adamec, Čenĕk und A. Satánek 1965. Teaching Machines and Health Education. International Journal of Health Education (8): 10-12.

Allegrante, John P., David A. Sleet und J. Michael McGinnis 2004. Mayhew Derryberry. Pioneer of Health Education. American Journal of Public Health (94:3): 370-371.

Antonovsky, Aaron und Hillei Shuvai 1966. Evaluation of the Effect of Mass Media in a Health Education Programme. International Journal of Health Education (9): 58-68.

Aronowitz, Robert A. 1998. Making Sense of Illness. Science, Society and Disease. Cambridge: Cambridge University Press.

Berlivet, Luc 2005. Uneasy prevention. The Problematic Modernisation of Health Education in France after 1975. In: Virginia Berridge und Kelly Loughlin (Hg.). Medicine, the Market and the Mass Media. Producing Health in the Twentieth Century. London u. New York: Routledge: 95-122.

Berlivet, Luc 2008. Between Expertise and Biomedicine: Public Health Research in France after the Second World War. Medical History (52:4): 471-492.

Berridge, Virginia 2006. The Policy Response to the Smoking and Lung Cancer Connection in the 1950s and 1960s. The Historical Journal (49:4): 1185-1209.

Berridge, Virginia 2007. Marketing Health. Smoking and the Discourse of Public Health in Britain, 1945-2000. Oxford, New York: Oxford University Press.

Berridge, Virginia 2010. Medizin, Public Health und die Medien in Großbritannien von 1950 bis 1980. In: Martin Lengwiler und Jeannette Madarász (Hg.). Das präventive Selbst. Eine Kulturgeschichte moderner Gesundheitspolitik. Bielefeld: transcript: 205-228.

Blackwell, Barbara 1964. Anticipated pre-medical Care Activities of upper middle-class Adults and their Implication for Health Education Practice. Health Education Monographs (1:17): 17-36.

Brückweh, Kerstin, Dirk Schumann, Richard F. Wetzell und Benjamin Ziemann (Hg.) 2012. Engineering Society. The Role of the Human and Social Sciences in Modern Societies, 1880-1980. Houndmills, Basingstoke, Hampshire, New York: Palgrave Macmillan.

Bruner, Jerome 1990. Acts of Meaning. Cambridge, MA: Harvard University Press.

Bundesminister für Gesundheitswesen (Käte Strobel) 1967. Erlaß über die Errichtung der Bundeszentrale für gesundheitliche Aufklärung vom 20. Juli 1967. Gemeinsames Ministerialblatt, 18, 374-375.

Bundesvereinigung für Gesundheitserziehung e.V. (Hg.) 1957-1986. Gesundheitserziehung von A-Z. Handbuch in Loseblattform. o. O.: o. V.

Bundesvereinigung für Gesundheitserziehung e.V. (Hg.) 1989. Bundesvereinigung für Gesundheitserziehung e. V. Ihre Entstehung, Ihre Aufgaben, Ihre Mitarbeiter. Bonn: B\&T Verlag.

Bundeszentrale für gesundheitliche Aufklärung (Hg.) 1970. Verhaltensbeeinflussung durch die Gesundheitserziehung. Methodologische Probleme. Beiträge zur Grundlagenforschung in der Gesundheitserziehung unterbreitet dem I. Internationalen Seminar für Gesundheitserziehung (Kommunikation, Intermediavergleich, Evaluation). Genf: International Journal of Health Education. 
Calver, Homer N. 1962. Letters to the Editor. A Commentary on „Development of Health Museums in the U.S.A.“. The Health Education Journal (20:4): 213-217.

Carlson, Eric T. und Robert I. Watson 1965. The Birth of a Journal. Journal of the History of the Behavioral Sciences (1:1): 3-4.

Dalton, James H., Maurice J. Elias und Abraham Wandersman 2007. Community Psychology. Linking Individuals and Communities. 2. Aufl. Belmont, CA: Thomson/Wadsworth.

Derryberry, Mayhew 1960a. Health Education-Its Objectives and Methods. Health Education Monographs (1:8): 1-9.

Derryberry, Mayhew 1960b. Research: Retrospective and Perspective. International Journal of Health Education (3:4): 164-169.

Deutscher Bundestag 24.1.1964. Plenarprotokoll der 109. Sitzung. 4. Wahlperiode (1961-1965). URL: http://dipbt.bundestag.de/doc/btp/04/04109.pdf (3.12.2018).

Deutscher Bundestag 11.3.1965. Plenarprotokoll der 171. Sitzung. 4. Wahlperiode (1961-1965). URL: http://dipbt.bundestag.de/doc/btp/04/04171.pdf (3.12.2018).

Eghigian, Greg 2015. The Corrigible and the Incorrigible. Science, Medicine, and the Convict in Twentieth-Century Germany. Ann Arbor, MI: University of Michigan Press.

Elliot, Rosemary 2010. From Youth Protection to Individual Responsibility. Adressing Smoking among Young People in Post-War West Germany. Medizinhistorisches Journal (45:1): 66-101.

Elliot, Rosemary 2012. Smoking for Taxes: The Triumph of Fiscal Policy over Health in Postwar West Germany, 1945-55. The Economic History Review (65:4): 1450-1474.

Elliot, Rosemary 2015. Inhaling Democracy: Cigarette Advertising and Health Education in Post-War West Germany, 1950s-1975. Social History of Medicine (28:4): 509-531.

Engelhardt, Roderich von und Martin Vogel 1930. Was will „Der Mensch“? In: Deutsches Hygiene-Museum und Martin Vogel (Hg.). Der Mensch. Vom Werden, Wesen und Wirken des menschlichen Organismus. Leipzig: Johann Ambrosius Barth: IV-V.

Etzemüller, Thomas (Hg.) 2009. Die Ordnung der Moderne. Social Engineering im 20. Jahrhundert. Bielefeld: transcript.

Ewbank, Alison J. 1966. Evaluation of the Mass Media-Television. Health Education Journal (25:3): 139-153.

Festinger, Leon 1978 [1957]. Theorie der kognitiven Dissonanz. Bern u.a.: Huber.

Fritsche, Wolfgang 1972. An Antismoking Campaign among Schoolchildren in Germany. In: Robert G. Richardson (Hg.). The Second World Conference on Smoking and Health. The Proceedings of a Conference organised by the Health Education Council at Imperial College, London, 20th to 24th September 1971. Bath: Pitman Medical: 77-80.

Fritsche, Wolfgang 1973. Bundesrepublik Deutschland. In: Bundeszentrale für gesundheitliche Aufklärung (Hg.). Gesundheitserziehung in Europa. Organisationsformen, Aktivitäten, Forschungsprojekte, Berufliche Ausbildung, Pläne für die Zukunft. Genf: Studer: 108-126.

Gastaldo, Denise 1997. Is Health Education good for You? Re-thinking Health Education through the Concept of Bio-Power. In: Alan Petersen und Robin Bunton (Hg.). Foucault, Health and Medicine. New York, NY: Routledge: 113-133.

Gebhard, Bruno 1962. Development of Health Museums in the U.S.A. An Attempt in Appraisal. The Health Education Journal (20:2): 87-97.

Glanz, Karen und Donald B. Bishop 2010. The Role of Behavioral Science Theory in Development and Implementation of Public Health Interventions. Annual Review of Public Health (31): 399-418. https://doi.org/10.1146/annurev.publhealth.012809.103604.

Griffiths, William 1957. Communication Problems facing our Profession. Health Education Monographs (1:1): 26-32.

Hagen, Wilhelm 1920. Hygienische Volksbelehrung. Entwurf eines Lehrplans und einer Organisation. Univ.-Diss., Hygienisches Institut der Universität Freiburg im Breisgau.

Hähner-Rombach, Sylvelyn (Hg.) 2015. Geschichte der Prävention. Akteure, Praktiken, Instrumente. Stuttgart: Franz Steiner.

Härtler, Gisela 2014. Statistisch gesichert und trotzdem falsch? Vom (Un-)Wesen statistischer Schlüsse. Berlin und Heidelberg: Springer Spektrum.

Hartley, Eugene L. 1961. Determinants of Health Beliefs and Behavior. I. Psychological Determinants. American Journal of Public Health (51:10): 1541-1547.

Heesen, Anke te 2012. Theorien des Museums zur Einführung. Hamburg: Junius. 
Heesen, Anke te und Mario Schulze 2015. Einleitung. In: Mario Schulze; Anke te Heesen und Vincent Dold (Hg.). Museumskrise und Ausstellungserfolg. Die Entwicklung der Geschichtsausstellung in den Siebzigern. Berlin: Druckerei der Humboldt-Universität zu Berlin: 7-17.

Hochbaum, Godfrey M. 1960. Research relating to Health Education. Health Education Monographs (1:8): 10-20.

Hochbaum, Godfrey M. 1965. Research to improve Health Education. International Journal of Health Education (8:3): 141-148.

Hopf, Ernst-Johannes (Hg.) 1976. Fünfundzwanzig Jahre Gesundheitsministerkonferenz in der Bundesrepublik Deutschland. Die Entschließungen der Gesundheitsministerkonferenz der Länder der Bundesrepublik Deutschland 1949-1974. Stuttgart: Kohlhammer.

Hünerbein, Josef 1953. Das öffentliche Gesundheitswesen in den Vereinigten Staaten von Nordamerika. Der öffentliche Gesundheitsdienst (15:1): 2-9 \& 49-55.

Institute für Gesundheitserziehung der sozialistischen Länder Europas (Hg.) 1980. Internationales Terminologisches Wörterbuch Gesundheitserziehung. Dresden: Institut für Gesundheitserziehung.

Kegeles, Stephen 1963. Some Problems in the Use of Mass Communication for Public Health. Health Education Journal (21:1): 29-35.

Keweloh, Hans-Walter 2016. Museen in der Bundesrepublik. In: Markus Walz (Hg.). Handbuch Museum. Geschichte, Aufgaben, Perspektiven. Stuttgart: J. B. Metzler: 65-69.

Krister, John S. 1967. The Mass Communicators and Health Issues: A Leadership Problem. International Journal of Health Education (10:4): 167-170.

Kunkel, Otto 1962. Das Deutsche Hygiene-Museum im Ausland. In: Deutsches HygieneMuseum Dresden (Hg.). 50 Jahre Deutsches Hygiene-Museum Dresden. Zentralinstitut für medizinische Aufklärung der Deutschen Demokratischen Republik. Dresden: Landesdruckerei Sachsen: 23-24.

Labisch, Alfons und Wolfgang Woelk 1998. Geschichte der Gesundheitswissenschaften. In: Klaus Hurrelmann und Ulrich Laaser (Hg.). Handbuch Gesundheitswissenschaften. Neuausgabe. Weinheim u. München: Juventa: 49-89.

Laukötter, Anja 2010. „Anarchie der Zellen“. Geschichte und Medien der Krebsaufklärung in der ersten Hälfte des 20. Jahrhunderts. URL: http://www.zeithistorische-forschungen. de/site/40208992/default.aspx (3.12.2018).

Laukötter, Anja 2018. Measuring Knowledge and Emotions: Audience Research in Educational Films at the Beginning of the Twentieth Century. In: Christian Bonah; David Cantor und Anja Laukötter (Hg.). Health Education Films in the Twentieth Century. Rochester, NY: University of Rochester Press: 305-326.

Lengwiler, Martin 2010. Konjunkturen und Krisen in der Verwissenschaftlichung der Sozialpolitik im 20. Jahrhundert. Archiv für Sozialgeschichte (50): 47-68.

Lengwiler, Martin und Jeannette Madarász (Hg.) 2010. Das präventive Selbst. Eine Kulturgeschichte moderner Gesundheitspolitik. Bielefeld: transcript.

Linek, Jenny 2016. Gesundheitsvorsorge in der DDR zwischen Propaganda und Praxis. Stuttgart: Franz Steiner.

Lingner, Karl August 1914. Der Mensch als Organisationsvorbild. Bern: Max Drechsel.

Lingner, Karl August 1930 [1904]. Einige Leitgedanken zu der Sonderausstellung Volkskrankheiten und ihre Bekämpfung. In: Julius Ferdinand Wollf (Hg.). Lingner und sein Vermächtnis. Hellerau: Verlag Jakob Hegner: 207-233.

Link, Fabian 2018. Sozialwissenschaften im Kalten Krieg: Mathematisierung, Demokratisierung und Politikberatung. URL: http://hsozkult.geschichte.hu-berlin.de/index.asp? $\mathrm{id}=3095 \& \mathrm{view}=\mathrm{pdf} \& \mathrm{pn}=$ forum \&type=forschungsberichte (3.12.2018).

Link, Jürgen 1997. Versuch über den Normalismus. Wie Normalität produziert wird. Opladen: Westdeutscher Verlag.

Ludwig, Werner 1973a. Deutsche Demokratische Republik. In: Bundeszentrale für gesundheitliche Aufklärung (Hg.). Gesundheitserziehung in Europa. Organisationsformen, Aktivitäten, Forschungsprojekte, Berufliche Ausbildung, Pläne für die Zukunft. Genf: Studer: 127-132.

Ludwig, Werner (Hg.) 1973b. Grundriß der Gesundheitserziehung. Berlin: VEB Verlag Volk und Gesundheit. 
Maasen, Sabine, Jens Elberfeld, Pascal Eitler und Maik Tändler (Hg.) 2011. Das beratene Selbst. Zur Genealogie der Therapeutisierung in den 〉langen〈Siebzigern. Bielefeld: transcript.

Madarász, Jeannette 2009. Gesellschaftliche Debatten um Krankheit: Das Risikofaktorenmodell zwischen Politik, Wirtschaft und Wissenschaft 1968-1986. Medizin, Gesellschaft und Geschichte (28): 187-211.

McLeary, Erin und Elizabeth Toon 2012. „Here Man Learns About Himself“: Visual Education and the Rise and Fall of the American Museum of Health. American Journal of Public Health (102:7): e27-e36. https://doi.org/10.2105/AJPH.2011.300560.

Mead, Margaret 1961. Determinants of Health Beliefs and Behavior. III. Cultural Determinants. American Journal of Public Health (51:10): 1552-1554.

Minister für Gesundheitswesen (Max Sefrin) 29.4.1967. Anordnung über das Statut des Deutschen Hygiene-Museums in der Deutschen Demokratischen Republik. Gesetzblatt der Deutschen Demokratischen Republik (Teil III/6): 39-41.

Münch, Richard 2002. Soziologische Theorie. Bd. 1: Grundlegung durch die Klassiker. Frankfurt am Main u. New York: Campus.

Münch, Richard 2004. Soziologische Theorie. Bd. 3: Gesellschaftstheorien. Frankfurt am Main u. New York: Campus.

Münch, Ragnhild und Jan Lazardzig 2002. Inszenierung von Einsicht und Überblick. Hygiene-Ausstellungen und Prävention. In: Sigrid Stöckel und Ulla Walter (Hg.). Prävention im 20. Jahrhundert. Historische Grundlagen und aktuelle Entwicklungen in Deutschland. Weinheim u. München: Juventa: 78-95.

Niehoff, Jens-Uwe und Ralf-Raigo Schrader 1991. Gesundheitsleitbilder - Absichten und Realitäten in der Deutschen Demokratischen Republik. In: Thomas Elkeles; Rolf Rosenbrock; Günter Ewert und Heinz-Harald Abolz (Hg.). Prävention und Prophylaxe. Theorie und Praxis eines gesundheitspolitischen Grundmotivs in zwei deutschen Staaten 1949-1990. Berlin: Edition Sigma: 51-74.

Nikolow, Sybilla 2015a. „Wissenschaftliches Stillleben“ des Körpers im 20. Jahrhundert. In: Sybilla Nikolow (Hg.). Erkenne Dich selbst! Strategien der Sichtbarmachung des Körpers im 20. Jahrhundert. Köln u. a.: Böhlau: 11-43.

Nikolow, Sybilla (Hg.) 2015b. Erkenne Dich selbst! Strategien der Sichtbarmachung des Körpers im 20. Jahrhundert. Köln u. a.: Böhlau.

Nikolow, Sybilla und Christine Brecht 2000. Displaying the Invisible. Volkskrankheiten on Exhibition in Imperial Germany. Studies in History and Philosophy of Biology and Biomedical Sciences (31:4): 511-530.

Nyswander, Dorothy B. 1961. Group Dynamics. Health Education Monographs (1:10): 3-15.

Nyswander, Dorothy B. 1967. The Open Society: Its Implications for Health Educators. Health Education Monographs (1:22): 3-15.

Osten, Philipp, Gabriele Moser, Christian Bonah, Alexandre Sumpf, Tricia Close-Koenig und Joël Danet, (Hg.) 2015. Das Vorprogramm. Lehrfilm, Gebrauchsfilm, Propagandafilm, unveröffentlichter Film in Kinos und Archiven am Oberrhein 1900-1970; eine französisch-deutsche Vergleichsstudie. Heidelberg, Strasbourg: A25 Rhinfilm.

Pfütsch, Pierre 2017. Das Geschlecht des „präventiven Selbst“. Prävention und Gesundheitsförderung in der Bundesrepublik Deutschland aus geschlechterspezifischer Perspektive (1949-2010). Stuttgart: Franz Steiner.

Pott, Elisabeth 2005. Die Bundeszentrale für gesundheitliche Aufklärung. Ihre Geschichte und Aufgaben. In: Thomas Deres (Hg.). Krank - gesund. 2000 Jahre Krankheit und Gesundheit in Köln. Köln: Kölnisches Stadtmuseum: 334-347.

Raphael, Lutz 1996. Die Verwissenschaftlichung des Sozialen als methodische und konzeptionelle Herausforderung für eine Sozialgeschichte des 20. Jahrhunderts. Geschichte und Gesellschaft (22:2): 165-193.

Reinecke, Christiane 2010a. Fragen an die sozialistische Lebensweise. Empirische Sozialforschung und soziales Wissen in der DDR „Fürsorgediktatur“. Archiv für Sozialgeschichte (50): 311-334.

Reinecke, Christiane 2010b. Wissensgesellschaft und Informationsgesellschaft, Version 1.0. Docupedia-Zeitgeschichte. URL: https://docupedia.de/zg/Wissensgesellschaft (3.12.2018). 
Reinecke, Christiane und Thomas Mergel (Hg.) 2012. Das Soziale ordnen. Sozialwissenschaften und gesellschaftliche Ungleichheit im 20. Jahrhundert. Frankfurt am Main: Campus.

Roeßiger, Susanne und Uta Schwarz (Hg.) 2011. Kamera! Licht! Aktion! Filme über Körper und Gesundheit 1915-1990. Dresden: Sandstein.

Rosen, George 1961. Evolving Trends in Health Education. Canadian Journal of Public Health (52:12): 499-506.

Rosenstock, Irwin M. 1960. Gaps and Potentials in Health Education Research. Health Education Monographs (1:8): 21-27.

Rosenstock, Irwin M. 1961. Decision-Making by Individuals. Health Education Monographs (1:11): $19-36$.

Rothstein, William G. 2008. Public Health and the Risk Factor. A History of an Uneven Medical Revolution. Rochester, NY: University of Rochester Press.

Rowntree, F. St. D. 1959. The Health Exhibition. The Health Education Journal (17:2): 109-118.

Ruckstuhl, Brigitte 2011. Gesundheitsförderung. Entwicklungsgeschichte einer neuen Public Health-Perspektive. Weinheim u. München: Juventa.

Sala, Roberto 2017. Verwissenschaftlichung des Sozialen - Politisierung der Wissenschaft? Zum Verhältnis von Wissenschaft und Politik in der Geschichtsschreibung des 19. und 20. Jahrhunderts. Berichte zur Wissenschaftsgeschichte (40:4): 333-349.

Sammer, Christian 2013. „Das Ziel ist das gesunde Leben!“. Die Verflechtungen zwischen dem Deutschen Gesundheits-Museum in Köln (DGM) und dem Deutschen HygieneMuseum in Dresden (DHM) in den 1950er Jahren. In: Detlev Brunner; Udo Grashoff und Andreas Kötzing (Hg.). Asymmetrisch verflochten? Neue Forschungen zur gesamtdeutschen Nachkriegsgeschichte. Berlin: Christoph Links: 131-145.

Sammer, Christian 2015. Die „Modernisierung“ der Gesundheitsaufklärung in beiden deutschen Staaten zwischen 1949 und 1975. Das Beispiel Rauchen. Medizinhistorisches Journal (50:3): 249-294.

Sarasin, Philipp 2011. Was ist Wissensgeschichte? Internationales Archiv für Sozialgeschichte der deutschen Literatur (36:1): 159-172. https://doi.org/10.1515/iasl.2011.010.

Schenk, Britta-Marie, Malte Thießen und Jan-Holger Kirsch (Hg.) 2013. Zeitgeschichte der Vorsorge. URL: http://www.zeithistorische-forschungen.de/3-2013 (3.12.2018).

Schmacke, Norbert 2002. Die Individualisierung der Prävention im Schatten der Medizin. In: Sigrid Stöckel und Ulla Walter (Hg.). Prävention im 20. Jahrhundert. Historische Grundlagen und aktuelle Entwicklungen in Deutschland. Weinheim u. München: Juventa: $178-189$.

Schnocks, Hans o. J. (ca. 1981). Die Geschichte der Bundeszentrale für gesundheitliche Aufklärung. Unveröffentlichtes Manuskript: Sammlung Deutsches Hygiene-Museum Dresden.

Schwartz, Friedrich Wilhelm 1995. Entwicklung von Public Health in Deutschland. Public Health Forum (3:10): 3-7.

Sorensen, Elaine Shaw 1987. Clair E. Turner: Pioneer in Health Education 1890-1974. Health Education (18:1): 37-39.

Steidle, Luitpold 1955. Zentralinstitut für Sanitär-Hygienische Aufklärung. In: Ministerium für Gesundheitswesen der Deutschen Demokratischen Republik (Hg.). Gesundheitsschutz in der Sowjetunion. Ergebnisse einer Studienreise deutscher Ärzte in die Sowjetunion. Berlin: VEB Verlag Volk und Gesundheit: 130-139.

Stein, Claudia 2013. Organising the History of Hygiene at the Internationale Hygiene-Ausstellung in Dresden in 1911. NTM (21:4): 355-387.

Steinmetz, Willibald 2003. Ungewollte Politisierung durch die Medien? Die Contergan-Affäre. In: Bernd Weisbrod (Hg.). Die Politik der Öffentlichkeit - Die Öffentlichkeit der Politik. Politische Medialisierung in der Geschichte der Bundesrepublik. Göttingen: Wallstein: $195-228$.

Steller, Thomas 2014. Volksbildungsinstitut und Museumskonzern. Das Deutsche HygieneMuseum 1912-1930. URL: http://d-nb.info/1070371769/34 (3.12.2018).

Stiftung Deutsches Hygiene-Museum (Hg.) 2001. „Rechtzeitig erkannt - heilbar“. Krebsaufklärung im 20. Jahrhundert. Dresden: Michel-Sandstein.

Stoff, Heiko 2015. Gift in der Nahrung. Zur Genese der Verbraucherpolitik Mitte des 20. Jahrhunderts. Stuttgart: Franz Steiner. 
Straschun, J. 1928. Die hygienische Volksaufklärung in der Union der Sozialistischen Sowjet-Republiken. Hygienischer Wegweiser. Zentralblatt für Technik und Methodik der hygienischen Volksbelehrung (3/3-4): 69.

Straus, Robert 1961. Determinants of Health Beliefs and Behavior. II. Sociological Determinants. American Journal of Public Health (51:10): 1547-1552.

Szöllösi-Janze, Margit 2004a. Wissensgesellschaft - ein neues Konzept zur Erschließung der deutsch-deutschen Zeitgeschichte? In: Hans Günter Hockerts (Hg.). Koordinaten deutscher Geschichte in der Epoche des Ost-West-Konflikts. München: R. Oldenbourg: 277-305.

Szöllösi-Janze, Margit 2004b. Wissensgesellschaft in Deutschland. Überlegungen zur Neubestimmung der deutschen Zeitgeschichte über Verwissenschaftlichungsprozesse. Geschichte und Gesellschaft (30:2): 277-313.

Tändler, Maik 2016. Das therapeutische Jahrzehnt. Der Psychoboom in den siebziger Jahren. Göttingen: Wallstein.

Tändler, Maik und Uffa Jensen 2012a. Psychowissen, Politik und das Selbst. Eine neue Forschungsperspektive auf die Geschichte des Politischen im 20. Jahrhundert. In: Maik Tändler und Uffa Jensen (Hg.). Das Selbst zwischen Anpassung und Befreiung. Psychowissen und Politik im 20. Jahrhundert. Göttingen: Wallstein: 9-35.

Tändler, Maik und Uffa Jensen (Hg.) 2012b. Das Selbst zwischen Anpassung und Befreiung. Psychowissen und Politik im 20. Jahrhundert. Göttingen: Wallstein.

Thießen, Malte 2017. Immunisierte Gesellschaft. Impfen in Deutschland im 19. und 20. Jahrhundert. Göttingen: Vandenhoeck \& Ruprecht.

Thränhardt, Rolf 1959. The German Hygiene Museum, Dresden. The Health Education Journal (17:2): 124-130.

Timmermann, Carsten 2010. Risikofaktoren: Der scheinbar unaufhaltsame Erfolg eines Ansatzes aus der amerikanischen Epidemiologie in der deutschen Nachkriegsmedizin. In: Martin Lengwiler und Jeannette Madarász (Hg.). Das präventive Selbst. Eine Kulturgeschichte moderner Gesundheitspolitik. Bielefeld: transcript: 251-277.

Tümmers, Henning 2017. AIDS. Autopsie einer Bedrohung im geteilten Deutschland. Göttingen: Wallstein Verlag.

Turner, Clair E. 1925. Malden Studies in Health Education. A Preliminary Report. American Journal of Public Health (15:5): 405-414.

Turner, Clair E. 1926. Personal und Community Health. 2. Aufl. Saint Louis: Mosby.

Turner, Clair E. 1928. Malden Studies on Health Education and Growth. American Journal of Public Health (18:10): 1217-1230.

Turner, Clair E. 1937. Personal Hygiene. Saint Louis: Mosby.

Turner, Clair E. 1952. Personal and Community Health. 9. Aufl. London: Henry Kimpton.

Turner, Clair E. 1964. World Progress and Problems in Health Education. International Journal of Health Education (7:3): 106-115.

Viborel, Lucien 1958a. How the Union was Born. International Journal of Health Education (1:2): 108-110.

Viborel, Lucien 1958b. What is the IUHEP? International Journal of Health Education (1:1): o. S.

Vogel, Martin 1925. Hygienische Volksbildung. In: Adolf Gottstein; Arthur Schlossmann und Ludwig Teleky (Hg.). Handbuch der Sozialen Hygiene und Gesundheitsfürsorge. Bd. 1: Grundlagen und Methoden. Berlin: Julius Springer: 303-390.

Weinert, Sebastian 2017. Der Körper im Blick. Gesundheitsausstellungen vom späten Kaiserreich bis zum Nationalsozialismus. Berlin und Boston: De Gruyter Oldenbourg.

Weischer, Christoph 2004. Das Unternehmen „Empirische Sozialforschung“. Strukturen, Praktiken und Leitbilder der Sozialforschung in der Bundesrepublik Deutschland. München: R. Oldenbourg.

Weisz, George 2014. Chronic Disease in the Twentieth Century. A History. Baltimore, Maryland: Johns Hopkins University Press.

Winkler, Anita 2015. Debating Sex: Education Films and Sexual Morality for the Young in Post-War Germany, 1945-1955. In: Christian Bonah und Anja Laukötter (Hg.). Screening diseases: Films on sex hygiene in Germany and France in the first half of the $20^{\text {th }}$ century. Gesnerus (72): 77-93. 
Young, Marjorie A. C. et al. (Hg.) 1963. Review of Research Related to Health Education Practice. Health Education Monographs (Supplement 1): iii-113.

Young, Marjorie A. C. et al. (Hg.) 1967-1969. Review of Research Related to Health Education Practice (1961-1966). Health Education Monographs (23-28).

Zander, Alvin 1962. Influencing People in the Face to Face Setting: Research Findings and their Application. Health Education Monographs (1:13): 3-17.

Ziemann, Benjamin 2012. Sozialgeschichte und Empirische Sozialforschung. Überlegungen zum Kontext und zum Ende einer Romanze. In: Pascal Maeder; Barbara Lüthi und Thomas Mergel (Hg.). Wozu noch Sozialgeschichte? Eine Disziplin im Umbruch. Festschrift für Josef Mooser zum 65. Geburtstag. Göttingen: Vandenhoeck \& Ruprecht: 131-149.

Zoller, Bernhard E. 1957. Gesundheitserziehung in Frankreich. Bericht über eine von der Welt-Gesundheitsorganisation gewährte Studienreise Mai-Juni 1954. Stuttgart: Georg Thieme.

\author{
Christian Sammer \\ Institut für Geschichte und Ethik der Medizin \\ Ruprecht-Karls-Universität Heidelberg \\ Im Neuenheimer Feld 327 \\ 69120 Heidelberg \\ Deutschland \\ christian.sammer@histmed.uni-heidelberg.de
}

